\title{
OFDM Carrier Synchronization Based on Time-Domain Channel Estimates
}

\author{
Hao Zhou, Amaresh V. Malipatil, and Yih-Fang Huang, Fellow, IEEE
}

\begin{abstract}
Carrier frequency synchronization is critical to the quality of signal reception in OFDM systems. This paper presents an approximate maximum-likelihood (ML) carrier frequency offset (CFO) estimation scheme based on time-domain channel estimates which retain the CFO information in the form of phase rotation. The proposed ML CFO estimate is investigated under static as well as time-varying fading channels. Statistical properties of the estimator are examined and Cramer-Rao lower bound (CRLB) is derived. Theoretical analysis and numerical simulations show that the proposed CFO estimator renders excellent performance with lower computational complexity. The proposed CFO estimate also has an advantage of allowing for more flexible pilot patterns.
\end{abstract}

Index Terms-Carrier synchronization, maximum likelihood estimate, OFDM, time-varying multipath fading channel.

\section{INTRODUCTION}

$\mathbf{T}$ HE demand for multi-media wireless communication services is pushing data rates up to hundreds of mega bits per second. At such high data rates, the physical nature of wireless channels are extremely time-varying and frequencyselective. Multi-carrier transmission is an effective way to combat such impairments [2], [3]. Orthogonal frequency division multiplexing (OFDM), one of the multi-carrier techniques, features high spectral efficiency and robustness to multipath. By transforming the wide-band frequency-selective channel into a set of narrow-band flat fading channels, OFDM receiver has a drastically simplified equalization process which can be accomplished using a one-tap frequency-domain equalizer. Therefore, OFDM has been employed in various commercial applications that include WLAN (IEEE 802.11a/g/n and HIPERLAN/2), WMAN (IEEE 802.16), DAB-T and DVB-T, and it is also considered a good candidate for the future $4 \mathrm{G}$ systems.

In OFDM systems, the number of subcarriers is typically in the order of hundreds, or even over thousands. These subcarriers are spaced close together in the frequency domain, and are supposed to be orthogonal to each other. As such, the synchronization requirement (which includes timing and

Manuscript received September 30, 2006; revised June 25, 2007 and May 20, 2008; accepted June 4, 2008. The associate editor coordinating the review of this paper and approving it for publication was $\mathrm{H}$. Li. This paper has been presented, in part, at the 2006 IEEE WCNC [1]. The technical content of the paper had been completed when Hao Zhou was a Ph.D. student in the Department of Electrical Engineering at the University of Notre Dame.

H. Zhou is with Atheros Communications, Santa Clara, CA 95054 (e-mail: haozhou_sh@hotmail.com).

A. V. Malipatil is with LSI Corp., Milpitas, CA 95035 (e-mail: amaresh191@yahoo.com).

Y.-F. Huang is with the University of Notre Dame, Notre Dame, IN 46556 (e-mail: huang@nd.edu).

Digital Object Identifier 10.1109/TWC.2008.060765. carrier frequency synchronization) for OFDM systems is more stringent than that for single carrier systems [4], [5], [6]. Much research has been done on this topic in the last two decades, see, e.g., [3], [7], [8], [9], [10].

This paper considers carrier frequency synchronization in OFDM systems. Carrier frequency offset (CFO) is attributable to two factors: one is the local oscillator frequency mismatch between the transmitter and the receiver; the other is channel Doppler spread, which is present in mobile environments due to changing channel conditions between the transmitter and the receiver. OFDM systems are sensitive to $\mathrm{CFO}$, because it causes inter-carrier interference (ICI) and attenuates the desired signal. These effects reduce the effective signal-tonoise ratio (SNR) in OFDM reception resulting in degraded system performance [11], [12].

In OFDM systems, carrier frequency synchronization is usually done in two steps. The first step is coarse synchronization, which usually reduces the CFO to within one-half of the subcarrier spacing [3]; this is followed by fine carrier synchronization, which further estimates and reduces the residual CFO. In this paper, we focus on the problem of fine carrier synchronization. For fine carrier synchronization, CFO estimation can be done in either the time domain [13] or the frequency domain [14], [8], [15]. Time-domain schemes are based on time-domain correlation of the received signal, while frequency-domain schemes are based on using the frequencydomain phase information in consecutive OFDM symbols. Most of the conventional fine carrier synchronization methods are frequency-domain approaches. In [8], Moose derived a maximum likelihood (ML) estimate for the CFO using two identical successive training symbols. In [15], Tsai et al. derived a weighted least-squares (WLS) method to estimate $\mathrm{CFO}$ and sampling clock offset. These methods do not take the channel temporal fading into account, so the estimation accuracy will degrade in the presence of Doppler fading.

This paper presents a CFO estimation scheme based on time-domain channel estimates. Time-domain channel estimation methods have been considered for OFDM systems by many researchers, see, e.g., [16], [17]. We show that timedomain channel estimates, such as the one using SVD in [17], retain the $\mathrm{CFO}$ information in the form of phase rotation of the estimated channel multipaths. This provides the motivation to design a CFO estimation scheme based on time-domain channel estimates, which turns out to be a simple maximum likelihood estimate (MLE) for the CFO, under some model approximations. Since it is always a function of the sufficient statistics, MLE makes the most efficient use of the information obtained from received data and usually renders very low error 
variance. In fact, it has been shown that if there exists an unbiased estimator whose error variance achieves the CramerRao lower bound (CRLB), the estimator is an MLE [18]. Incorporating the Doppler effect of the fading channel, the proposed CFO MLE is shown to achieve performance gain over existing schemes [8], [15] under time-varying fading channels.

Performance evaluation of the proposed scheme is made through theoretical analysis as well as numerical simulations. The Cramer-Rao lower bound for the CFO estimate is derived for both static and fading scenarios. All the results show that the proposed CFO estimation scheme has excellent performance with considerably reduced complexity. The complexity reduction is due to the fact that the proposed estimator is derived from the time-domain channel estimates whose length is usually much shorter compared to the Fast Fourier Transform (FFT) size. Furthermore, the proposed CFO estimation scheme has a distinct advantage of allowing for more flexibility in pilot design.

The paper is organized as follows. The system model is presented in Section II. In Section III, the CFO estimation scheme is first derived for the static scenario, and then extended to the fading scenario. CRLB is derived in Section IV for both static and fading scenarios. In Section V, statistical characteristics (particularly the mean and the variance) of the estimates are analyzed. Section VI presents simulation results to compare the performance of the proposed scheme to other CFO estimators. Some concluding remarks are given in Section VII.

\section{SySTEM MODEL}

\section{A. OFDM modulation and wireless channel}

At the transmitter, the $i^{\text {th }}$ OFDM symbol generated from the frequency domain symbols $\underline{X}_{i}=\left[X_{i, 0}, X_{i, 1}, \cdots, X_{i, N-1}\right]^{T}$ can be formulated as

$$
\underline{x}_{i}=\frac{1}{N} \mathbf{W}^{H} \underline{X}_{i}
$$

where $N$ is the FFT size, $\mathbf{W}$ is the $N \times N$ FFT matrix with $[\mathbf{W}]_{k n}=e^{j 2 \pi k n / N}$ and $\underline{x}_{i}=\left[x_{i, 0}, x_{i, 1}, \cdots, x_{i, N-1}\right]^{T}$ is the resulting time-domain OFDM symbol. A cyclic prefix (CP) of length $N_{g}$ is usually added to avoid the inter-symbol interference (ISI) and to help retain the orthogonality of the subcarriers in multipath fading scenario.

A typical multipath fading channel is considered here. This channel consists of $L$ uncorrelated paths, each of which is characterized by a fixed path delay $\tau_{l}$ and a time-varying complex path amplitude $\alpha_{i, l}$. The time-varying multipath channel during the $i^{t h}$ symbol can be characterized by an impulse response function as [19]

$$
h_{i}(\tau)=\sum_{l=1}^{L} \alpha_{i, l} \delta\left(\tau-\tau_{l}\right)
$$

The baseband OFDM signal (1) is converted to an analog waveform, up-converted to radio frequency (RF) and transmitted through the time-varying multipath channel (2). The signal is corrupted by additive white Gaussian noise (AWGN). At the receiver, perfect timing is assumed throughout this paper.
After down-conversion, sampling and removal of the $\mathrm{CP}$, the received signal is stored in a length- $N$ data vector $\underline{r}_{i}$.

If the carrier synchronization is perfect, after $\underline{r}_{i}$ is transformed into frequency domain by FFT, the frequency domain symbol $\underline{S}_{i}$ is obtained, namely,

$$
\underline{S}_{i}=\mathbf{W} \underline{r}_{i}=\mathbf{X}_{i} \underline{H}_{i}+\underline{Z}_{i}
$$

where, $\mathbf{X}_{i}$ is the diagonal data matrix with diagonal entries $\left[\mathbf{X}_{i}\right]_{k, k}=X_{i, k}, \underline{Z}_{i}$ is the frequency domain noise with $\underline{Z}_{i} \sim$ $\mathcal{C N}\left(0, N \sigma^{2} \mathbf{I}\right)$ and $\underline{H}_{i}$ is the channel transfer function (CTF) whose elements can be expressed as [19]

$$
H_{i, n}=\sum_{l=1}^{L} \alpha_{i, l} \exp \left[-j \frac{2 \pi n \tau_{l}}{T}\right]
$$

where $1 / T$ is the subcarrier spacing.

In the presence of a normalized CFO $\epsilon=\Delta f T$, where $\Delta f$ is the actual CFO, the received signal will be

$$
y_{i, n}=r_{i, n} \cdot e^{j \frac{2 \pi \epsilon}{N}\left[(i-1)\left(N+N_{g}\right)+N_{g}+n\right]}
$$

The frequency domain symbol $\underline{Y}_{i}$ after FFT is given by

$$
\underline{Y}_{i}=\mathbf{W} \underline{y}_{i}=\mathbf{W} \mathbf{D}_{i} \underline{r}_{i}
$$

where $\mathbf{D}_{i}$ is the $N \times N$ diagonal matrix with diagonal elements given by

$$
\left[D_{i}\right]_{n, n}=e^{j \frac{2 \pi \epsilon}{N}\left[(i-1)\left(N+N_{g}\right)+N_{g}+n\right]}, 0 \leq n \leq N-1
$$

Furthermore,

$$
\underline{Y}_{i}=\mathbf{W D}_{i} \underline{\underline{r}}_{i}=\underbrace{\frac{1}{N} \mathbf{W D}_{i} \mathbf{W}^{H}}_{\mathbf{D}_{i}^{\prime}} \mathbf{W}_{\underline{r}_{i}}=\mathbf{D}_{i}^{\prime}\left[\mathbf{X}_{i} \underline{H}_{i}+\underline{Z}_{i}\right]
$$

The symbol index $i$ may be omitted subsequently whenever it is obvious, for elegance of representation.

\section{B. Successive OFDM symbol reception under $C F O$}

The conventional way to estimate CFO considers the phase rotation between two successive OFDM symbols. From (8), the outputs of the FFT for the $i^{t h}$ and the $(i+1)^{t h}$ symbols are, respectively,

$$
\begin{aligned}
\underline{Y}_{i} & =\mathbf{D}_{i}^{\prime} \mathbf{x}_{i} \underline{H}_{i}+\mathbf{D}_{i}^{\prime} \underline{Z}_{i} \\
\underline{Y}_{i+1} & =\mathbf{D}_{i+1}^{\prime} \mathbf{x}_{i+1} \underline{H}_{i+1}+\mathbf{D}_{i+1}^{\prime} \underline{Z}_{i+1}
\end{aligned}
$$

Assume that the same OFDM symbols are repeated, i.e., $\mathbf{X}_{i+1}=\mathbf{X}_{i}=\mathbf{X}$. Noting that $\mathbf{D}_{i+1}{ }^{\prime}=e^{j \alpha \epsilon} \mathbf{D}_{i}^{\prime}$, where $\alpha=$ $2 \pi\left(N+N_{g}\right) / N, \underline{Y}_{i+1}$ can be expressed in terms of $\underline{Y}_{i}$ as follows:

$$
\begin{aligned}
\underline{Y}_{i+1} & =e^{j \alpha \epsilon} \underline{Y}_{i}+e^{j \alpha \epsilon} \mathbf{D}_{i}^{\prime} \mathbf{X}\left(\underline{H}_{i+1}-\underline{H}_{i}\right) \\
& +\mathbf{D}_{i+1}^{\prime}\left(\underline{Z}_{i+1}-\underline{Z}_{i}\right)
\end{aligned}
$$

Here $\underline{Z}_{i+1}$ and $\underline{Z}_{i}$ are uncorrelated noise terms. The channel $\underline{H}_{i}$ and noise $\underline{Z}_{i}$ are assumed to be uncorrelated. Note that in the presence of Doppler fading, our model will not consider the time varying effect inside one OFDM symbol, but $\underline{H}_{i}$ and $\underline{H}_{i+1}$ in (11) are correlated. More specifically, $\underline{H}_{i}$ is modeled as a complex Gaussian process and its time-domain correlation 


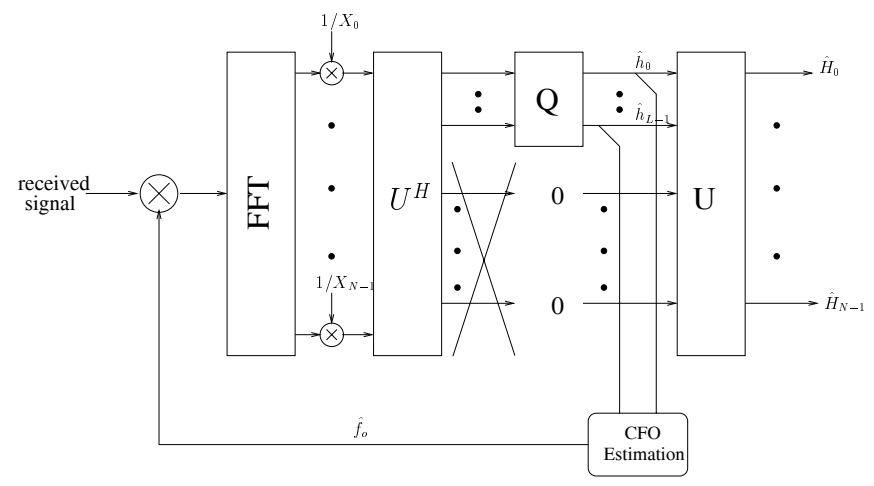

Fig. 1. OFDM receiver structure with time domain channel estimate and the proposed CFO estimation.

function depends on the Doppler frequency $F_{d}$ and is given as [19]

$$
R_{t, n}=J_{0}\left(2 \pi n F_{d} T_{s}\right)
$$

where $J_{0}(\cdot)$ is a Bessel function of the first kind of order 0 [20] and $T_{s}$ is the OFDM symbol period. Here, $n$ is the index of the OFDM symbol.

The frequency-domain correlation function of $\underline{H}_{i}$ depends on the uncorrelated multipath (2) and can be expressed as [19]

$$
R_{f, k}=\sum_{l=1}^{L} \sigma_{l}^{2} \exp \left[-j \frac{2 \pi k \tau_{l}}{T}\right]
$$

where $\sigma_{l}^{2}$ is the average power of the $l^{\text {th }}$ path, and the sum of $\sigma_{l}^{2}$ is normalized to one throughout this paper.

Note that, in the case that the channel remains unchanged for successive OFDM symbols, namely, a static channel $\left(\underline{H}_{i+1}=\underline{H}_{i}\right)$ [8], (11) would reduce to

$$
\underline{Y}_{i+1}=e^{j \alpha \epsilon} \underline{Y}_{i}+\mathbf{D}_{i+1}^{\prime}\left(\underline{Z}_{i+1}-\underline{Z}_{i}\right)
$$

\section{Time-domain successive channel estimates under $\mathrm{CFO}$}

In this section, we present an idea of using the time-domain channel estimates (instead of the frequency domain symbol $\left.\underline{Y}_{i}\right)$ for CFO estimation. A time-domain channel estimation method has been proposed by Beek and Edfors [16] [17] and has been extended to two dimensional channel estimation in [21]. Here we employ the method based on singular-value decomposition (SVD) presented in [17] due to its simplicity in theoretical analysis. In the SVD-based OFDM channel estimation, optimal rank reduction can be achieved by using the SVD of the channel frequency-domain autocovariance, namely,

$$
\mathbf{R}_{\underline{H H}}=\mathbf{U} \Lambda \mathbf{U}^{H}
$$

where $\mathbf{R}_{\underline{H H}}$ is the frequency-domain autocovariance consisting of $\left\{\overline{R_{f, k}}\right\}, \Lambda$ is the diagonal matrix of singular values $\left(\lambda_{k}\right)$, $\mathbf{U}$ is the unitary matrix with the singular vectors of $\mathbf{R}_{\underline{H H}}$ as its columns. The corresponding receiver architecture is shown in Fig. 1 [16].

To begin, consider the modified rank- $p$ least-squares (LS) frequency-domain channel transfer function (CTF) estimate, which is given by [16]

$$
\underline{\hat{H}}_{L S}=\mathbf{U} \Delta_{T} \mathbf{U}^{H} \mathbf{X}^{-1} \underline{Y}
$$

where, $\Delta_{T}$ is an $N \times N$ diagonal matrix with diagonal elements

$$
\left[\Delta_{T}\right]_{k, k}= \begin{cases}1, & k=1,2, \cdots, p \\ 0, & k=p+1, \cdots, N\end{cases}
$$

Since $\mathbf{U}$ is unitary, the intermediate result in (16), $\mathbf{U}^{H} \mathbf{X}^{-1} \underline{Y}$, yields a time-domain estimate with uncorrelated components. The diagonal matrix $\Delta_{T}$ truncates the estimated time-domain channel impulse response (CIR) to retain only the $p$ most dominant multipaths.

The intermediate length- $p$ time-domain CIR is obtained as follows [17].

$$
\underline{\hat{h}}_{i}=\mathbf{U}_{p}^{H} \mathbf{X}^{-1} \underline{Y}_{i}=\mathbf{U}_{p}^{H} \mathbf{X}^{-1} \mathbf{D}_{i}^{\prime}\left[\mathbf{X}_{i} \underline{H}_{i}+\underline{Z}_{i}\right]
$$

The $N \times p$ matrix $\mathbf{U}_{p}$ is formed by the first $p$ columns of $\mathbf{U}$.

Premultiplying (11) by $\mathbf{U}_{p}^{H} \mathbf{X}^{-1}$, the subsequent timedomain channel estimate under the influence of $\mathrm{CFO}$ can be deduced as,

$$
\begin{aligned}
\underline{\hat{h}}_{i+1} & =\underline{e}^{j \alpha \epsilon} \underline{\hat{h}}_{i}+\underbrace{\mathbf{U}_{p}^{H} \mathbf{X}^{-1} \mathbf{D}_{i+1}^{\prime} \mathbf{X}\left(\underline{H}_{i+1}-\underline{H}_{i}\right)}_{\text {I }} \\
& +\underbrace{\mathbf{U}_{p}^{H} \mathbf{X}^{-1} \mathbf{D}_{i+1}^{\prime}\left(\underline{Z}_{i+1}-\underline{Z}_{i}\right)}_{\text {II }}
\end{aligned}
$$

In the special case of a static channel, i.e., $\underline{H}_{i+1}=\underline{H}_{i}$, (19) reduces to

$$
\underline{\hat{h}}_{i+1}=e^{j \alpha \epsilon} \underline{\hat{h}}_{i}+\mathbf{U}_{p}^{H} \mathbf{X}^{-1} \mathbf{D}_{i+1}^{\prime}\left(\underline{Z}_{i+1}-\underline{Z}_{i}\right)
$$

On the other hand, if we consider the optimal rank- $p$ frequency-domain CTF estimate, i.e., the MMSE estimate,

$$
\underline{\hat{H}}_{M M S E}=\mathbf{U} \Delta \mathbf{U}^{H} \mathbf{X}^{-1} \underline{Y}
$$

where, $\Delta$ is a diagonal matrix with elements

$$
[\Delta]_{k, k}= \begin{cases}\frac{\lambda_{k}}{\lambda_{k}+\beta / \gamma}, & k=1,2, \cdots, p \\ 0, & k=p+1, \cdots, N\end{cases}
$$

$\gamma=\frac{E\left\{|X|^{2}\right\}}{N \sigma^{2}}$ is the average SNR per subcarrier. Apart from providing the MMSE estimate, the diagonal matrix $\Delta$ here also truncates the time-domain channel response to retain only the $p$ most dominant multipaths.

The intermediate MMSE time-domain CIR is thus given by [17],

$$
{\underline{\hat{h}_{o p}}}_{i}=\Delta_{p} \mathbf{U}_{p}^{H} \mathbf{X}^{-1} \underline{Y}_{i}=\Delta_{p} \mathbf{U}_{p}^{H} \mathbf{X}^{-1} \mathbf{D}_{i}^{\prime}\left[\mathbf{X}_{i} \underline{H}_{i}+\underline{Z}_{i}\right]
$$

where the $p \times p$ matrix $\Delta_{p}$ is formed by the first $p$ rows and columns of $\Delta$.

Similarly, considering two successive OFDM symbols gives

$$
\begin{aligned}
{\underline{h_{o p}}}_{i+1} & =e^{j \alpha \epsilon}{\underline{h_{o p}}}_{i}+\underbrace{\Delta_{p} \mathbf{U}_{p}^{H} \mathbf{X}^{-1} \mathbf{D}_{i+1}^{\prime} \mathbf{X}\left(\underline{H}_{i+1}-\underline{H}_{i}\right)}_{\mathrm{I}} \\
& +\underbrace{\Delta_{p} \mathbf{U}_{p}^{H} \mathbf{X}^{-1} \mathbf{D}_{i+1}^{\prime}\left(\underline{Z}_{i+1}-\underline{Z}_{i}\right)}_{\text {II }}
\end{aligned}
$$

For the special case of a static channel, it reduces to

$$
\underline{\hat{h}}_{o p}=e^{j \alpha \epsilon} \underline{\hat{h}}_{o p}+\Delta_{p} \mathbf{U}_{p}^{H} \mathbf{X}^{-1} \mathbf{D}_{i+1}^{\prime}\left(\underline{Z}_{i+1}-\underline{Z}_{i}\right)
$$


From (19), (20), (24) and (25), one can see that using the SVD-based channel estimates reduces the dimension of the signal space from the number of subcarriers to the number of effective multipaths while retaining the CFO information $(\epsilon)$ which appears as the phase rotation of estimated channel multipaths. This is the key idea of the proposed CFO estimate in this paper, i.e., using the phase rotation of the multipath channel estimates to estimate the CFO.

\section{Time Domain MaXimum LiKelihood CFO ESTIMATE}

\section{A. Maximum Likelihood CFO estimate under static channel}

We derive the ML CFO estimate using the static channel model (20). To obtain the MLE for the CFO, the conditional probability density function $f\left(\underline{\hat{h}}_{p_{i+1}} ; \epsilon \mid \underline{h}_{p_{i}}\right)$ is considered. It is evident from (20) that $f\left({\underline{\hat{h}_{p}}}_{i+1} ; \epsilon \mid \underline{\hat{h}}_{i}\right)$ is Gaussian with mean and covariance given by

$$
\begin{aligned}
& E\left[\underline{\hat{h}}_{i+1} \mid \underline{\hat{h}}_{i}\right]=e^{j \alpha \epsilon} \underline{\hat{h}}_{i} \\
& \mathbf{C}_{{\underline{\hat{h}_{p}}}_{i+1} \underline{\hat{h}}_{\underline{h}_{i}}}=2 N \sigma^{2} \mathbf{U}_{p}^{H} \mathbf{X}^{-1}\left[\mathbf{X}^{-1}\right]^{H} \mathbf{U}_{p} \approx \frac{2 \beta}{\gamma} \mathbf{I}
\end{aligned}
$$

The approximation $\mathbf{X}^{-1}\left[\mathbf{X}^{-1}\right]^{H} \approx E\left\{1 /|X|^{2}\right\} \mathbf{I}=$ $\beta / E\left\{|X|^{2}\right\} \mathbf{I}$, where, $\beta=E\left\{|X|^{2}\right\} E\left\{1 /|X|^{2}\right\}$ is a constellation dependent factor, is used to obtain (27). Note that for PSK modulation, (27) is exactly equal without any approximation. For QAM signals, it will be shown later that this approximation simplifies the MLE derivation, though it leads to some performance loss.

Henceforth, the maximum likelihood CFO estimate is obtained by setting the first derivative of the log-likelihood function to zero, namely,

$$
\frac{\partial}{\partial \epsilon} \ln \left\{f\left(\underline{\hat{h}}_{i+1} ; \epsilon \mid \underline{\hat{h}}_{i}\right)\right\}=0
$$

and the MLE is given by

$$
\left.\hat{\epsilon}_{M L E}=\frac{1}{\alpha}\left(\angle \underline{h}_{\underline{h}}^{H}{ }_{i} \underline{h}_{p+1}\right]\right)
$$

As mentioned at the end of Section II, intuitively, when CFO is present, each multipath of the channel estimate undergoes a common phase rotation as time progresses. The proposed maximum likelihood CFO estimator then takes the phase difference between two successive channel estimates to obtain the estimate.

On the other hand, if we use the MMSE channel estimates in (25) to derive the maximum likelihood CFO estimate, the conditional mean and covariance of $f\left(\underline{\hat{h}}_{o p} ; \epsilon \mid \underline{\hat{h}}_{o p}\right)$ is given by

$$
\begin{aligned}
& E\left[\underline{\hat{h}}_{o p} i+1 \underline{h}_{o p}\right]=e^{j \alpha \epsilon} \underline{\hat{h}}_{o p} i \\
& \mathbf{C}_{{\underline{\hat{h}_{o p}}}_{i+1} \underline{\hat{h}_{o p}}}=2 N \sigma^{2} \Delta_{p} \mathbf{U}_{p}^{H} \mathbf{X}^{-1}\left[\mathbf{X}^{-1}\right]^{H} \mathbf{U}_{p} \Delta_{p}^{H} \approx \frac{2 \beta}{\gamma} \Delta_{p}^{2} \mathbf{I}
\end{aligned}
$$

The maximum likelihood $\mathrm{CFO}$ estimation is given accordingly as

$$
\begin{aligned}
\hat{\epsilon}_{M L E} & =\frac{1}{\alpha}\left(\angle\left[\underline{\hat{h}}_{o p}{ }_{i}^{H} \mathbf{C}_{\underline{\hat{h}}_{o p}^{-1}} \underline{\underline{h}}_{i+1} \underline{\hat{h}}_{o p} i \underline{\hat{h}}_{o p}{ }_{i+1}\right]\right) \\
& =\frac{1}{\alpha}\left(\angle\left[\underline{\hat{h}}_{i}{ }^{H}{\underline{\hat{h}_{p}}}_{i+1}\right]\right)
\end{aligned}
$$

It is interesting to see from (32) that the ML CFO estimate provided by the MMSE channel estimates has the same formulation as that provided by the LS channel estimates. The same result also holds true in the fading channel case.

\section{B. Maximum Likelihood CFO estimate under fading channel}

Next we consider CFO estimation with a fading channel model. Based on the preceding time-domain channel estimate model (19) and using the complex Gaussian fading channel statistics described in Section II-B, we can show that $f\left(\hat{h}_{p_{i+1}} ; \epsilon \mid \underline{h}_{p_{i}}\right)$ is Gaussian with mean and covariance given below for the time-varying fading scenario (see Appendix I).

$$
\begin{aligned}
E\left[\underline{\hat{h}}_{i+1} \mid \underline{\hat{h}}_{i}\right] & \approx e^{j \alpha \epsilon} a \Lambda_{p}\left(\Lambda_{p}+\frac{\beta}{\gamma} \mathbf{I}\right)^{-1} \underline{\hat{h}}_{\underline{p}} \\
& \approx e^{j \alpha \epsilon} \underline{\hat{h}}_{p} \\
\mathbf{C}_{{\underline{h_{p}}}_{i+1} \mid \underline{\hat{h}}_{i}} & \approx\left(\Lambda_{p}+\frac{\beta}{\gamma} \mathbf{I}\right)-a^{2} \Lambda_{p}^{2}\left(\Lambda_{p}+\frac{\beta}{\gamma} \mathbf{I}\right)^{-1} \\
& \approx \frac{2 \beta}{\gamma}\left(\frac{\gamma(1-a)}{\beta} \Lambda_{p}+\mathbf{I}\right)
\end{aligned}
$$

where, according to the time-selective channel model (12), $a=J_{0}\left(2 \pi F_{d} T_{s}\right)$ is the fading coefficient, and $\Lambda_{p}$ is the $p \times p$ diagonal matrix consisting of the $p$ most dominant eigenvalues in $\Lambda$. Here we use the same approximation for $\mathbf{X}^{-1}\left[\mathbf{X}^{-1}\right]^{H}$ as in the static channel. Additionally, $\mathbf{D}_{i+1}{ }^{\prime}=e^{j \alpha \epsilon i} \mathbf{D}_{1}^{\prime}$. In a closed-loop operation, the CFO estimate is fed back to correct the carrier frequency. Consequently, the residual CFO remains small, thus $\mathbf{D}_{1}^{\prime} \approx \mathbf{I}$. Besides, from (33) to (34) and from (35) to (36), we assume that $a$ is close to 1 and SNR is in the medium to high range. The final covariance matrix is seen to be independent of time index $i$. The MLE is obtained from (28) as below.

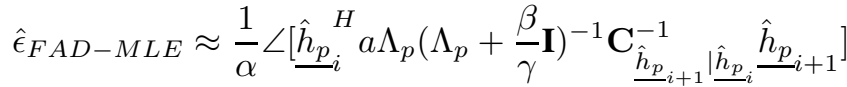

$$
\begin{aligned}
& \approx \frac{1}{\alpha} \angle\left[\underline{\hat{h}}_{i}^{H} \mathbf{C}_{\underline{\hat{h}}_{p_{i+1}}^{-1}}^{-1} \underline{\hat{h}}_{\underline{p}} \underline{\hat{h}}_{i+1}\right]
\end{aligned}
$$

Note that the matrix inversion in (37) is simple and acts as a weighting vector for the inner product of $\underline{h}_{p}$ and $\underline{h}_{p}$, because $\mathbf{C}_{\underline{h}_{\underline{p}}} \underline{\underline{h}}_{i+1} \underline{\underline{h}}_{i}$ in (36) is a diagonal matrix.

As for the MMSE channel-estimate-based ML CFO estimate, we can obtain an equivalent result as

$$
\begin{aligned}
& \hat{\epsilon}_{F A D-M L E} \approx \frac{1}{\alpha} \angle\left[\underline{\hat{h}}_{o p}^{H}{ }_{i}^{H} \mathbf{C}_{\underline{\hat{h}}_{o p}}^{-1}{ }_{i+1} \underline{h}_{\underline{h}^{\prime}} \underline{h}^{\frac{\hat{h}_{o p}}{i+1}}\right] \\
& \left.=\frac{1}{\alpha} \angle \underline{\underline{h}}_{\underline{p}}^{H} \mathbf{C}_{{\underline{\hat{h}_{p}}}_{i+1} \mid \underline{h}_{i}}^{-1} \underline{\hat{h}}_{i+1}\right]
\end{aligned}
$$

where the covariance matrix is given by

$$
\mathbf{C}_{{\underline{\hat{h}_{o p}}}_{i+1} \underline{\hat{h}_{o p}} i} \approx \frac{2 \beta}{\gamma} \Delta_{p}^{2}\left(\frac{\gamma(1-a)}{\beta} \Lambda_{p}+\mathbf{I}\right)
$$

Compare the MLE derived for the fading channel (37) with that for the static channel (29), the term inside the angle expression of (37) is the weighted inner product of the estimated channel while the term inside (29) can be seen as a simple inner product with equal weight. We rewrite the formula as (40) and will refer to this estimate as equal-weight 
estimator for the purpose of comparison in the following sections.

$$
\left.\hat{\epsilon}_{M L E}=\frac{1}{\alpha}\left(\angle \underline{\underline{h}}_{p}^{H} \underline{\hat{h}}_{p} \underline{-1+1}_{i+1}\right]\right)
$$

Figure 1 shows a schematic diagram of the OFDM receiver structure with the proposed CFO estimator. The ' $Q$ ' block varies depending on whether the LS or the MMSE channel estimate is used.

\section{CRAMER-RAo Lower Bound}

In this section, we derive the CRLB under static channel and time-varying fading channel.

\section{A. Static Case}

The observation equations (9) and (10) are repeated here as,

$$
\begin{aligned}
\underline{Y}_{i} & =\mathbf{D}_{i}^{\prime} \mathbf{X} \underline{H}+\mathbf{D}_{i}^{\prime} \underline{Z}_{i}=\mathbf{D}_{i}^{\prime} \underline{S}+\mathbf{D}_{i}^{\prime} \underline{Z}_{i} \\
\underline{Y}_{i+1} & =\mathbf{D}_{i+1}^{\prime} \underline{\mathbf{X}} \underline{H}+\mathbf{D}_{i+1}^{\prime} \underline{Z}_{i+1}=\mathbf{D}_{i+1}^{\prime} \underline{S}+\mathbf{D}_{i+1}^{\prime} \underline{Z}_{i+1}
\end{aligned}
$$

Here, $\underline{S}=\mathbf{X} \underline{H}$ and the channel is assumed to be static, i.e., $\underline{H}_{i}=\underline{H}_{i+1}=\underline{H}$. A vector of deterministic and unknown parameters is defined as

$$
\underline{M}=\left[\epsilon|\underline{S}|^{T} \angle \underline{S}^{T}\right]^{T}
$$

The probability density function (pdf) of $\underline{Y}=\left[\underline{Y}_{i}^{T} \underline{Y}_{i+1}^{T}\right]^{T}$ is $\mathcal{C N}\left(\mu_{\underline{Y}}, \mathbf{C}_{\underline{Y}}\right)$. where,

$$
\mu_{\underline{Y}}=\left[\left(\mathbf{D}_{i}^{\prime} \underline{S}\right)^{T}\left(\mathbf{D}_{i+1}^{\prime} \underline{S}\right)^{T}\right]^{T}
$$

and $\mathbf{C}_{\underline{Y}}=N \sigma^{2} \mathbf{I}_{2 N \times 2 N}$. The Fischer information matrix (FIM) [22] is calculated as follows.

$$
\begin{aligned}
{\left[\mathbf{F I M}_{\underline{M}}\right]_{i, j} } & =2 \cdot \operatorname{Re}\left\{\frac{\partial \mu_{\underline{Y}}^{H}}{\partial \underline{M}_{i}} \mathbf{C}_{\underline{Y}}^{-1} \frac{\partial \mu_{\underline{Y}}}{\partial \underline{M}_{j}}\right\} \\
& +\operatorname{tr}\left\{\mathbf{C}_{\underline{Y}}^{-1} \frac{\partial \mathbf{C}_{\underline{Y}}}{\partial \underline{M}_{i}} \mathbf{C}_{\underline{Y}}^{-1} \frac{\partial \underline{\mathbf{C}}_{\underline{Y}}}{\partial \underline{M}_{j}}\right\}
\end{aligned}
$$

Here, only the estimation of $\epsilon$ is considered, thus only $\operatorname{CRLB}(\epsilon)=\left[\mathbf{F I M}_{\underline{M}}^{-1}\right]_{1,1}$ is evaluated. $\mathbf{C}_{\underline{Y}}$ is independent of $\underline{M}$. Also,

$$
\frac{\partial \mu_{\underline{Y}}}{\partial \epsilon}=\left[\frac{\frac{1}{N} \mathbf{W D}_{i} \mathbf{E}_{i} \mathbf{W}^{H} \underline{S}}{\frac{1}{N} \mathbf{W D}_{i+1} \mathbf{E}_{i+1} \mathbf{W}^{H} \underline{S}}\right]
$$

where, $\mathbf{E}_{i}$ is a diagonal matrix with diagonal elements $\left[\mathbf{E}_{i}\right]_{n, n}=j \frac{2 \pi}{N}\left\{(i-1)\left(N+N_{g}\right)+N_{g}+n\right\}$. Thus,

$$
\left[\mathbf{F I M}_{\underline{M}}\right]_{1,1}=\frac{2}{N \sigma^{2}}\left\{\frac{1}{N} \underline{S}^{H} \mathbf{W}\left(\mathbf{E}_{i}^{H} \mathbf{E}_{i}+\mathbf{E}_{i+1}^{H} \mathbf{E}_{i+1}\right) \mathbf{W}^{H} \underline{S}\right\}
$$

$$
\underbrace{\left[\mathbf{F I M}_{\underline{M}}\right]_{1, n}}_{n=2,3 \cdots, N+1}=\frac{2}{N \sigma^{2}} \operatorname{Re}\left\{\frac{1}{N} \underline{S}^{H} \mathbf{W}\left(\mathbf{E}_{i}+\mathbf{E}_{i+1}\right) \mathbf{W}^{H} \mathbf{F}_{n} e^{j \angle \underline{S}}\right\}
$$$$
\underbrace{\left[\mathbf{F I M}_{\underline{M}}\right]_{1, n}}_{n=N+2, \cdots, 2 N+1}=\frac{2}{N \sigma^{2}} \operatorname{Re}\left\{\frac{j}{N} \underline{S}^{H} \mathbf{W}\left(\mathbf{E}_{i}+\mathbf{E}_{i+1}\right) \mathbf{W}^{H} \mathbf{F}_{n} \underline{S}\right\}
$$

$$
\begin{aligned}
& \underbrace{\left[\mathbf{F I M}_{\underline{M}}\right]_{n, n}}_{n=2,3 \cdots, N+1}=\frac{2}{N \sigma^{2}} \cdot 2 \\
& \underbrace{\left[\mathbf{F I M}_{\underline{M}}\right]_{n, n}}_{n=N+2, \cdots, 2 N+1}=\frac{2}{N \sigma^{2}} \cdot 2|\underline{S}|_{n}^{2} \\
& \underbrace{\left[\mathbf{F I M}_{\underline{M}}\right]_{m, n}}_{n \neq m, n>1 \& m>1}=0
\end{aligned}
$$

where $\left[\mathbf{F}_{n}\right]_{n, n}=1$ and all the other elements are zero. The $\operatorname{FIM}_{\underline{M}}$ is of the form,

$$
\mathbf{F I M}_{\underline{M}}=\left[\begin{array}{cc}
d & \underline{b}^{T} \\
\underline{b} & \mathbf{K}
\end{array}\right]
$$

where, $d=\left[\mathbf{F I M}_{\underline{M}}\right]_{1,1}, \underline{b}^{T}=\left[\mathbf{F I M}_{\underline{M}}\right]_{1,2: 2 N+1}$ and $\mathbf{K}=$ $\operatorname{diag}\left\{\left[\mathbf{F I M}_{\underline{M}}\right]_{n, n}, \bar{n}=2,3, \cdots 2 N+\overline{1\}}\right.$ is a diagonal matrix. As noted before $\mathbf{C R L B}(\epsilon)=\left[\mathbf{F I M}_{\underline{M}}^{-1}\right]_{1,1}=(d-$ $\left.\underline{b}^{T} \mathbf{K}^{-1} \underline{b}\right)^{-1}$. After some algebraic manipulation, using the fact that, $\left[\mathbf{F}_{n}\right]\left[\mathbf{F}_{m}\right]=0$ for $n \neq m$, the $\mathbf{C R L B}(\epsilon)$ is given by

$$
\begin{aligned}
\operatorname{CRLB}(\epsilon) & =\frac{1}{\alpha^{2}} \frac{N \sigma^{2}}{|\underline{S}|^{2}} \approx \frac{1}{\alpha^{2}} \frac{N \sigma^{2}}{E\left[|X|^{2}\right] \underline{H}^{H} \underline{H}} \\
& =\frac{1}{\alpha^{2} \gamma} \frac{1}{\underline{H}^{H} \underline{H}}=\frac{1}{\alpha^{2} \gamma N}
\end{aligned}
$$

\section{B. Fading Case}

Under the fading channel assumption, the system is modelled as

$$
\begin{aligned}
\underline{Y}_{i} & =\mathbf{D}_{i}^{\prime} \mathbf{X} \underline{H}_{i}+\mathbf{D}_{i}^{\prime} \underline{Z}_{i} \\
\underline{Y}_{i+1} & =\mathbf{D}_{i+1}^{\prime} \mathbf{X} \underline{H}_{i+1}+\mathbf{D}_{i+1}^{\prime} \underline{Z}_{i+1}
\end{aligned}
$$

where, $\underline{H}_{i}, \underline{H}_{i+1} \sim \mathcal{C N}\left(\underline{0}, \mathbf{R}_{\underline{H} H}\right)$ are samples of the complex Gaussian process during symbols $i$ and $i+1$, respectively. Furthermore, due to Doppler fading, $\underline{H}_{i}$ and $\underline{H}_{i+1}$ are correlated; i.e., $E\left[\underline{H}_{i} \underline{H}_{i+1}^{H}\right]=E\left[\underline{H}_{i+1} \underline{H}_{i}^{H}\right]=a \mathbf{R}_{\underline{H H}}$. The joint pdf of 
$\underline{Y}$ is then,

$$
\begin{aligned}
f(\underline{Y} ; \epsilon) & \sim \mathcal{C N}\left(\underline{0}_{2 N \times 1}, \mathbf{C}_{\underline{Y}}\right) \\
\mathbf{C}_{\underline{Y}} & =\left[\begin{array}{c|c}
\mathbf{D}_{1}^{\prime}\left(\mathbf{P}+N \sigma^{2} \mathbf{I}\right) \mathbf{D}_{1}^{\prime H} & a e^{-j \alpha \epsilon} \mathbf{D}_{1}^{\prime} \mathbf{P D}_{1}^{\prime H} \\
\hline a e^{j \alpha \epsilon} \mathbf{D}_{1}^{\prime} \mathbf{P} \mathbf{D}_{1}^{\prime H} & \mathbf{D}_{1}^{\prime}\left(\mathbf{P}+N \sigma^{2} \mathbf{I}\right) \mathbf{D}_{1}^{\prime H}
\end{array}\right] \\
& =\left[\begin{array}{c|c|c}
\mathbf{D}_{1}^{\prime} & \mathbf{0}_{N \times N} \\
\hline \mathbf{0}_{N \times N} & \mathbf{D}_{1}^{\prime}
\end{array}\right]\left[\begin{array}{c|c}
\mathbf{P}+N \sigma^{2} \mathbf{I} & a e^{-j \alpha \epsilon} \mathbf{P} \\
\hline a e^{j \alpha \epsilon} \mathbf{P} & \mathbf{P}+N \sigma^{2} \mathbf{I}
\end{array}\right] \\
& \times\left[\begin{array}{c|c}
\mathbf{D}_{1}^{\prime H} & \mathbf{0}_{N \times N} \\
\hline \mathbf{0}_{N \times N} & \mathbf{D}_{1}^{\prime H}
\end{array}\right] \\
& \text { with } \mathbf{P}=\mathbf{X R}_{\underline{H H}} \mathbf{X}^{H} .
\end{aligned}
$$

In the fading case, the deterministic but unknown parameter vector reduces to a scalar, i.e., $\epsilon$. Since $E[\underline{Y}]=\underline{0}$, from (43), the FIM which is a scalar is given by,

$$
\mathbf{F I M}_{\epsilon}=\operatorname{tr}\left\{\mathbf{C}_{\underline{Y}}^{-1} \frac{\partial \mathbf{C}_{\underline{Y}}}{\partial \epsilon} \mathbf{C}_{\underline{Y}}^{-1} \frac{\partial \mathbf{C}_{\underline{Y}}}{\partial \epsilon}\right\}
$$

The CRLB for the fading channel is given accordingly as

$$
\mathbf{C R L B}(\epsilon)=\mathbf{F I M}_{\epsilon}^{-1}=1 / \operatorname{tr}\left\{\mathbf{C}_{\underline{Y}}^{-1} \frac{\partial \mathbf{C}_{\underline{Y}}}{\partial \epsilon} \mathbf{C}_{\underline{Y}}^{-1} \frac{\partial \mathbf{C}_{\underline{Y}}}{\partial \epsilon}\right\}
$$

When the $\mathrm{CFO} \epsilon$ is small, under the assumption $\mathbf{D}_{1}^{\prime}=$ $\mathbf{I}_{N \times N}, \mathbf{C}_{\underline{Y}}$ can be simplified to

$$
\mathbf{C}_{\underline{Y}}=\left[\begin{array}{c|c}
\mathbf{P}+N \sigma^{2} \mathbf{I} & a e^{-j \alpha \epsilon} \mathbf{P} \\
\hline a e^{j \alpha \epsilon} \mathbf{P} & \mathbf{P}+N \sigma^{2} \mathbf{I}
\end{array}\right]=\left[\begin{array}{c|c}
\mathbf{A} & \mathbf{B} \\
\hline \mathbf{C} & \mathbf{D}
\end{array}\right]
$$

Note that, this assumption actually removes the CFO information inside one OFDM symbol, but it helps to obtain an approximated CRLB for the model that only considers CFO effect between two successive OFDM symbols under fading channels. The derivative of $\mathbf{C}_{Y}$ in (58) can be derived as (Appendix B)

$$
\frac{\partial \mathbf{C}_{\underline{Y}}}{\partial \epsilon}=\mathbf{Q C}_{\underline{Y}}-\mathbf{C}_{\underline{Y}} \mathbf{Q}, \quad \text { where } \mathbf{Q}=\left[\begin{array}{c|c}
\mathbf{0} & \mathbf{0} \\
\hline \mathbf{0} & j \alpha \mathbf{I}
\end{array}\right]
$$

Thus, the FIM would be,

$$
\mathbf{F I M}_{\epsilon}=2 \cdot \operatorname{tr}\left(\mathbf{Q Q}-\mathbf{Q C}_{\underline{Y}}^{-1} \mathbf{Q C}_{\underline{Y}}\right)
$$

which can be simplified as,

$$
\mathbf{F I M}_{\epsilon}=2 \alpha^{2} \cdot \operatorname{tr}\left\{\mathbf{D}^{-1} \mathbf{C S}_{\mathbf{c}}{ }^{-1} \mathbf{B}\right\}=2 \alpha^{2} \cdot \operatorname{tr}\left\{\mathbf{A S}_{\mathbf{c}}{ }^{-1}-\mathbf{I}\right\}
$$

where, $\mathbf{S}_{\mathbf{c}}=\mathbf{A}-\mathbf{B D}^{-1} \mathbf{C}$ is the Schur's complement in the inversion of block matrix $\mathbf{C}_{\underline{Y}}$.

The trace expression can be further simplified when $\mathbf{X}^{-1}=$ $\mathbf{X}^{H}$, which assumes that the signal constellation has a constant envelope like Phase Shift Keying (PSK) or 4-QAM signal. The final result shows that (Appendix B)

$$
\mathbf{F I M}_{\epsilon} \approx 2 \alpha^{2} E\left[\left|X_{i}\right|^{2}\right] \operatorname{tr}\left\{\mathbf{R}_{\underline{H H}}^{\prime}\right\}
$$

where, $\mathbf{R}_{\underline{H H}}^{\prime}=\mathbf{U}\left\{\left(\Lambda+N \sigma^{2} \mathbf{I}\right)\left[\Lambda+N \sigma^{2} \mathbf{I}-a^{2} \Lambda(\Lambda+\right.\right.$ $\left.\left.N \sigma^{2} \mathbf{I}\right)^{-1} \frac{1+}{\Lambda]^{-1}}-\mathbf{I}\right\} \mathbf{U}^{H}$. Though the derivation of (63) assumes a constant envelop signal constellation, numerical simulations shows that the exact CRLB (62) and the approximate CRLB (63) match closely, even for the non-constant envelope constellations such as 64-QAM.

\section{Mean and Variance Analysis}

In this section, we analyze the mean and error variance of the proposed time-domain ML CFO estimate. Following the derivation in Section III, we consider a general channel model that accommodates both (19) and (20), which can be simply formulated as

$$
\underline{\hat{h}}_{i+1}=e^{j \alpha \epsilon} \underline{\hat{h}}_{i}+\underline{w}_{i+1}
$$

where, $\underline{w}_{i+1} \sim \mathcal{C N}\left(\underline{0}, \mathbf{C}_{{\underline{\hat{h}_{p}}}_{i+1}{\underline{\hat{h}_{p}}}_{i}}\right)$. For high SNR, the CFO estimation error can be approximated by (Appendix C)



It is easily seen from above that the proposed estimator is an unbiased estimator, i.e., $E\left[\hat{\epsilon}-\epsilon \mid \hat{h}_{p_{i}}\right]=0$.

For error variance analysis, $\overline{w e}$ first consider the static channel, in which, $\mathbf{C}_{{\underline{\hat{h}_{p}}}_{i+1} \mid \underline{\hat{h}}_{p_{i}}} \approx \frac{2 \beta}{\gamma} \mathbf{I}$, (65) can be simply formulated as

$$
\hat{\epsilon}-\epsilon \approx \frac{1}{\alpha}\left\{\frac{\operatorname{Im}\left[\left(\underline{h}_{p} e^{j \alpha \epsilon}\right)^{H} \underline{w}_{i+1}\right]}{{\underline{h_{p}}}_{i}{ }^{H}{\underline{h_{p}}}_{i}}\right\}
$$

Then the variance of the estimation error is (Appendix C)

$$
\operatorname{var}(\hat{\epsilon}-\epsilon) \approx \frac{1}{\alpha^{2}} \cdot \frac{\beta}{\gamma} \cdot \frac{1}{E\left[\underline{h}_{\underline{h_{p}}}^{H}{\underline{h_{p}}}_{i}\right]}=\frac{1}{\alpha^{2}} \cdot \frac{\beta}{N \gamma}
$$

Comparing with the CRLB for the static channel (52), this result shows that, at high SNRs, the error variance of the proposed MLE differs from the CRLB by a scaling factor $\beta$. For PSK signal, $\beta=1$, the error variance of the MLE achieves the CRLB. For QAM signal, in general $\beta \neq 1$, the performance loss of the MLE relative to the CRLB is due to the approximation of $\mathbf{C}_{\hat{h}_{p}}{ }_{i+1} \hat{h}_{\underline{p}}$ in (27) as $\frac{2 \beta}{\gamma} \mathbf{I}$ for the ML estimator. Intuitively, by taking the expectation on the signal $\mathbf{X}$, the ML estimator will lose certain performance compared with the optimal combination such as the Moose MLE, which uses the received signal $\underline{Y}=\mathbf{X} \underline{H}+\underline{Z}$ (exact $\underline{Y}$ without any approximation on $\mathbf{X}$ ). As we can see, the WLS method [15] uses the same approximation for the signal $\mathbf{X}$ in its derivation, therefore it suffers the same constellation pattern related performance loss, which is verified in the simulations (Fig. 5) in Section VI. However, by using this approximation, both the proposed MLE and the WLS scheme have the advantage that they can work even under different successive symbols.

As for time-varying channels, $\mathbf{C}_{{\underline{\hat{h}_{p}}}_{i+1} \mid \underline{\hat{h}}_{\underline{i}}} \approx \frac{2 \beta}{\gamma}\left(\frac{\gamma(1-a)}{\beta} \Lambda_{p}+\right.$ I). At high SNR, (65) can be written as (Appendix C)

$$
\hat{\epsilon}-\epsilon \approx \frac{1}{\alpha}\left\{\frac{\operatorname{Im}\left[\left(\underline{h}_{i} e^{j \alpha \epsilon}\right)^{H}\left(\frac{2 \beta}{\gamma}\left(\frac{\gamma(1-a)}{\beta} \Lambda_{p}+\mathbf{I}\right)\right)^{-1} \underline{w}_{i+1}\right]}{{\underline{h_{p}}}_{i}^{H}\left(\frac{2 \beta}{\gamma}\left(\frac{\gamma(1-a)}{\beta} \Lambda_{p}+\mathbf{I}\right)\right)^{-1}{\underline{h_{p}}}_{i}}\right\}
$$

The variance of the estimation error is derived as (Appendix C)

$$
\operatorname{var}\left(\hat{\epsilon}-\epsilon \mid \underline{\hat{h}}_{\underline{p}}\right) \approx \frac{1}{\alpha^{2}} \cdot \frac{\beta}{\gamma \sum_{k=1}^{p} \frac{\lambda_{k}}{\frac{\gamma}{\beta}(1-a) \lambda_{k}+1}}
$$


TABLE I

COST207 12-Multipath Typical Urban (TU) Channel Model

\begin{tabular}{|c|c|c|c|c|c|c|}
\hline Path Delay $(\mu \mathrm{s})$ & 0 & 0.1 & 0.3 & 0.5 & 0.8 & 1.1 \\
\hline Ave. Power $(\mathrm{dB})$ & -4.0 & -3.0 & 0 & -2.6 & -3.0 & -5.0 \\
\hline Path Delay $(\mu \mathrm{s})$ & 1.3 & 1.7 & 2.3 & 3.1 & 3.2 & 5.0 \\
\hline Ave. Power $(\mathrm{dB})$ & -7.0 & -5.0 & -6.5 & -8.6 & -11.0 & -10.0 \\
\hline
\end{tabular}

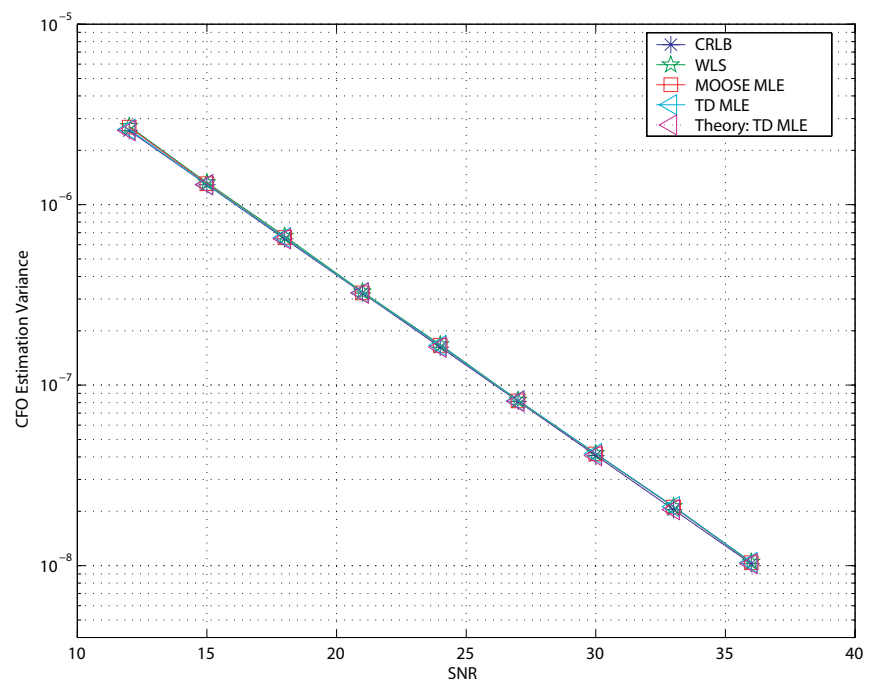

Fig. 2. Performance comparison of various CFO estimation schemes under static channel conditions: same PSK pilot symbols.

It will be interesting to compare the error variance of the equal-weight estimator (40) with the MLE (37) under the fading channel. The estimation error of the equal-weight estimator would be

$$
\hat{\epsilon}-\epsilon \approx \frac{1}{\alpha}\left\{\frac{\operatorname{Im}\left[\left(\underline{h}_{\underline{p}} e^{j \alpha \epsilon}\right)^{H} \underline{w}_{i+1}\right]}{{\underline{h_{p}}}_{i}^{H}{\underline{h_{p}}}_{i}}\right\}
$$

Its error variance turns out to be

$$
\begin{aligned}
& \operatorname{var}(\hat{\epsilon}-\epsilon) \approx \frac{1}{\alpha^{2}} \cdot \frac{\beta}{\gamma} \cdot \frac{E\left[{\underline{h_{p}}}_{i}^{H}\left(\frac{\gamma(1-a)}{\beta} \Lambda_{p}+\mathbf{I}\right){\underline{h_{p}}}_{i}\right]}{\left.E\left[{\underline{\left(h_{p}\right.}}_{i}{ }^{H}{\underline{h_{p}}}_{i}\right)^{2}\right]} \\
& =\frac{1}{\alpha^{2}} \cdot \frac{N \beta+\sum_{k=1}^{p} \lambda_{k}^{2} \gamma(1-a)}{\gamma N^{2}}
\end{aligned}
$$

We can see from the simulation results in the next session that the equal-weight estimator has larger error variance compared to the proposed MLE under fading channels.

\section{Simulation Results and Analysis}

Computer simulations were performed with the following system parameters: number of subcarriers $N=512$, length of cyclic prefix $N_{g}=52$, normalized CFO (frequency deviation / subcarrier spacing) $f_{o}=0.01$. The Jakes model [20] was employed to simulate the multipath channel with $L=12$ dominant components under static and fading scenarios as in the classical COST207 Typical Urban (TU) model (Table I). According to the channel model discussed in Section II, we applied the Jake's model with channel taps varying every symbol. The sample variance of the CFO estimation error was calculated for 400 OFDM symbols and the results were

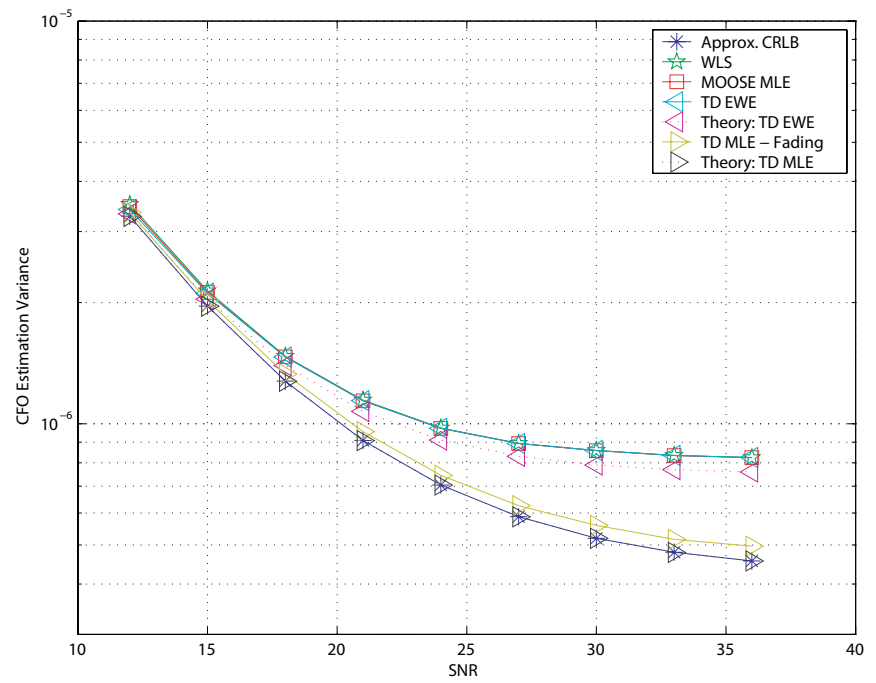

Fig. 3. Performance comparison of various CFO estimation schemes under slowly fading channel conditions: same PSK pilot symbols and $F_{d}=0.005$.

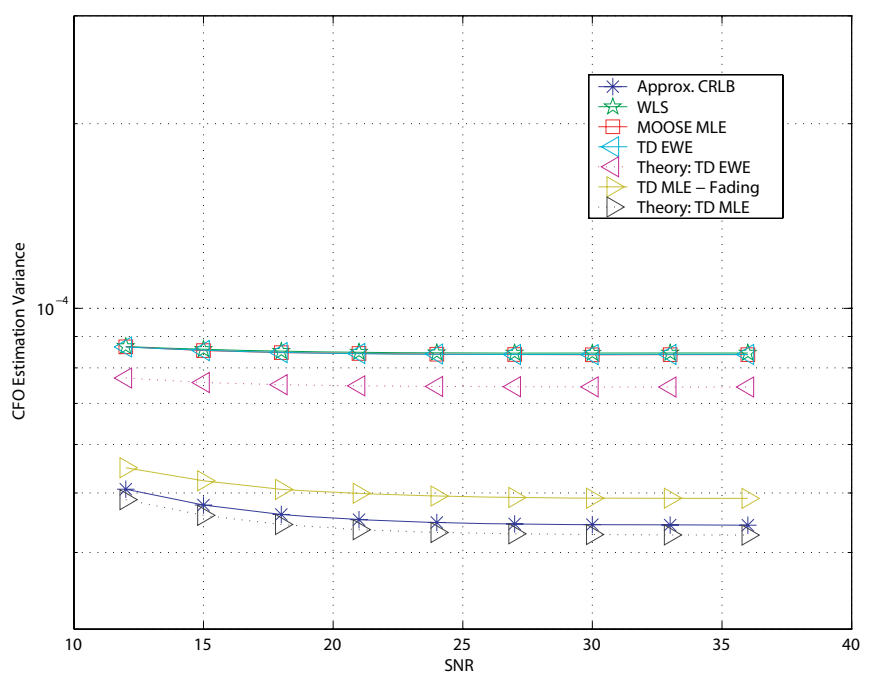

Fig. 4. Performance comparison of various CFO estimation schemes under fast fading channel conditions: same PSK pilot symbols and $F_{d}=0.05$.

averaged over 1000 Monte-Carlo simulations for each SNR value. The performance of the proposed estimator was compared with the scheme proposed by Moose [8] and frequencydomain WLS approach presented in [15].

We first investigated the proposed CFO estimator for PSK pilots. Repeated successive BPSK OFDM pilots were used in the simulation. In Fig. 2, we show the comparison of the error variance of the proposed scheme with those of WLS, Moose MLE, and with the derived CRLB, under static channel conditions. In this case, all estimators had almost identical performance with error variance approaching the CRLB. Fig. 3 and Fig. 4 show the performance comparison under slow and fast channel fading, respectively. For both cases, the timedomain MLE has a performance gain over other schemes and its variance is closest to the CRLB. In Fig. 4 (fast fading), the theoretical error variance of the TD MLE is lower than the CRLB. This is due to the approximation taken in the error variance derivation for the fading channel. By comparing the time-domain MLE (TD MLE) to the equal-weight time- 


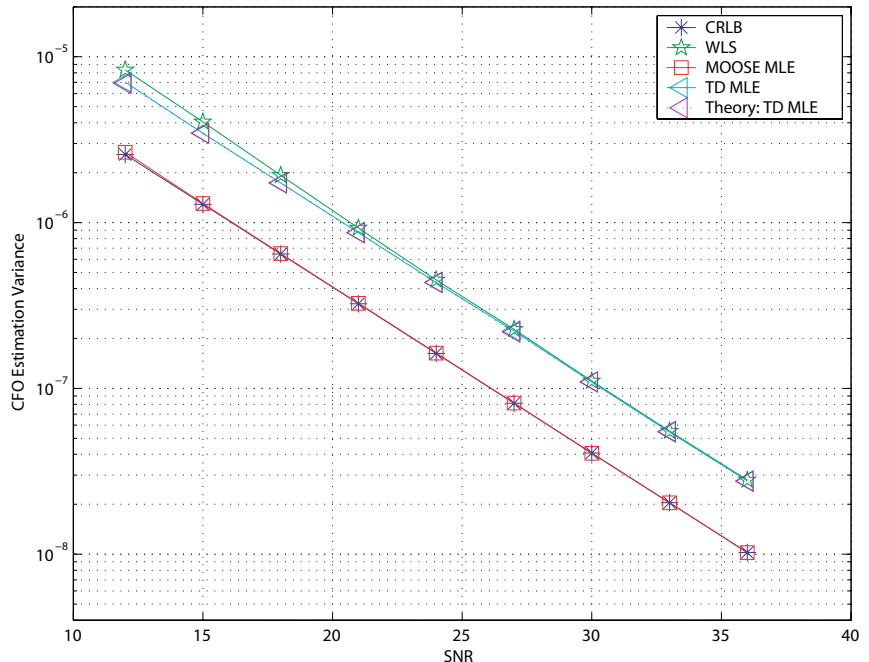

Fig. 5. Performance comparison of various CFO estimation schemes under static channel conditions: same QAM pilot symbols.

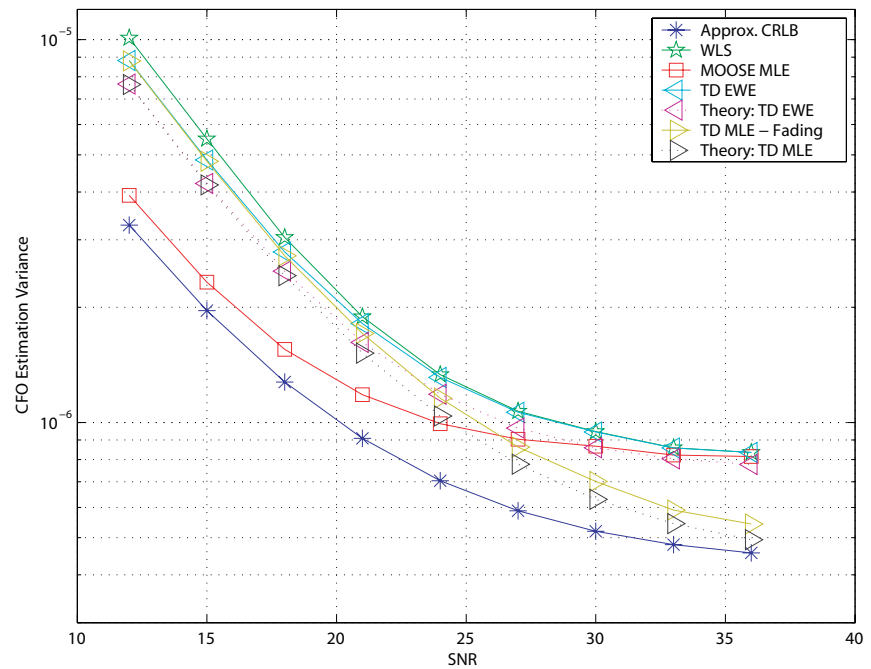

Fig. 6. Performance comparison of various CFO estimation schemes under slowly fading channel conditions: same QAM pilot symbols and $F_{d}=0.005$.

domain estimator (TD EWE) (40), it is easily seen that the performance gain comes from the weighting coefficients related to the channel Doppler.

We next considered the proposed CFO estimator with QAM pilots. Figures 5, 6 and 7 show performance comparison under static, slow and fast fading cases, respectively. By using identical successive QAM OFDM pilots, the time-domain CFO estimate suffered some performance degradation due to the approximation in (27). Under static channel condition, the performance loss due to the approximation (27) for the QAM symbol is equal to $\beta$, which is consistent with the error variance analysis given in Section V. It shows that the performance loss using (27) actually depends on the signal constellation. For the 64QAM modulation, $\beta \cong 2.68$ which is about $4 \mathrm{~dB}$. We can observe this loss by comparing the performance of the Moose MLE (which does not suffer from this approximation loss) to that of the proposed time-domain schemes in Fig. 5. In the fading cases, this performance loss will be partly recovered by the fading gain of the proposed MLE. For slow fading channels (Fig. 6), the Moose MLE

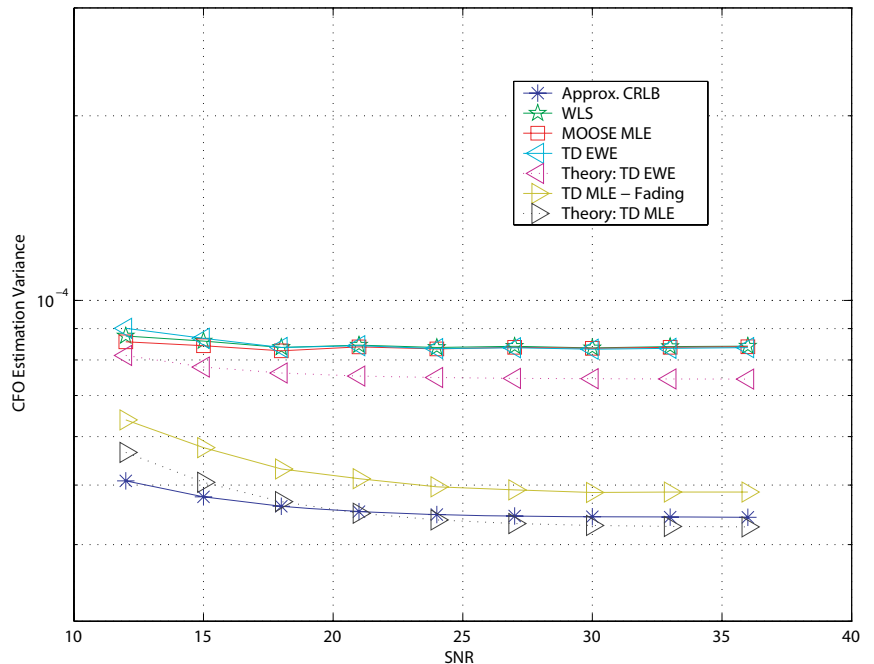

Fig. 7. Performance comparison of various CFO estimation schemes under fast fading channel conditions: same QAM pilot symbols and $F_{d}=0.05$.

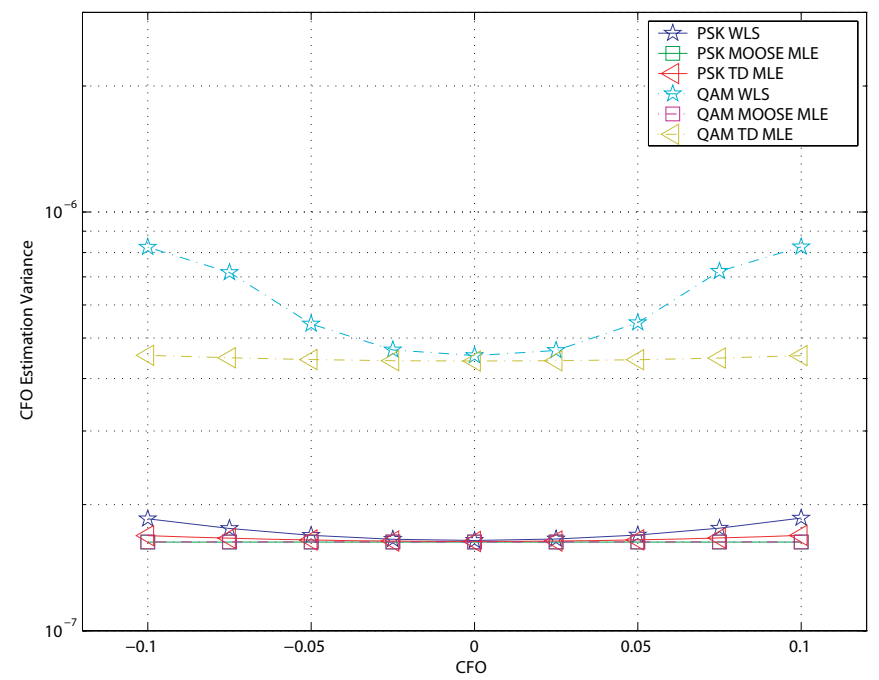

Fig. 8. Performance of various CFO estimation schemes under different CFO levels: static channels and $\mathrm{SNR}=24 \mathrm{~dB}$.

performs better than the proposed scheme at low SNRs (below $26 \mathrm{~dB}$ ) and is then surpassed by the time-domain MLE at higher SNR. For fast fading channels (Fig. 7), the time-domain MLE is always better than the Moose scheme in the interested SNR range due to significant fading gain.

It is easily seen from (11) and (19) that the estimation range of the proposed scheme is $[-\pi / \alpha, \pi / \alpha]$, which is approximately equal to $\pm \frac{1}{2}$ of the carrier spacing. Here we investigate the performance of the algorithm for different $\mathrm{CFO}$ levels inside the estimation range and compare with other algorithms. The simulation results are shown in Figures 8 and 9 for SNR equal to $24 \mathrm{~dB}$. For a static channel, the Moose scheme is least sensitive to the CFO while the WLS scheme appears to be most sensitive to large CFO values especially for QAM modulation. Also notice that two Moose MLE curves overlapped in Fig. 8, which verifies that the performance of the Moose MLE under the static channel is independent of the signal constellation. In Fig. 9, results of the same investigations are provided for both slow fading (lower set of six curves) and fast fading (upper set of six curves). Note 


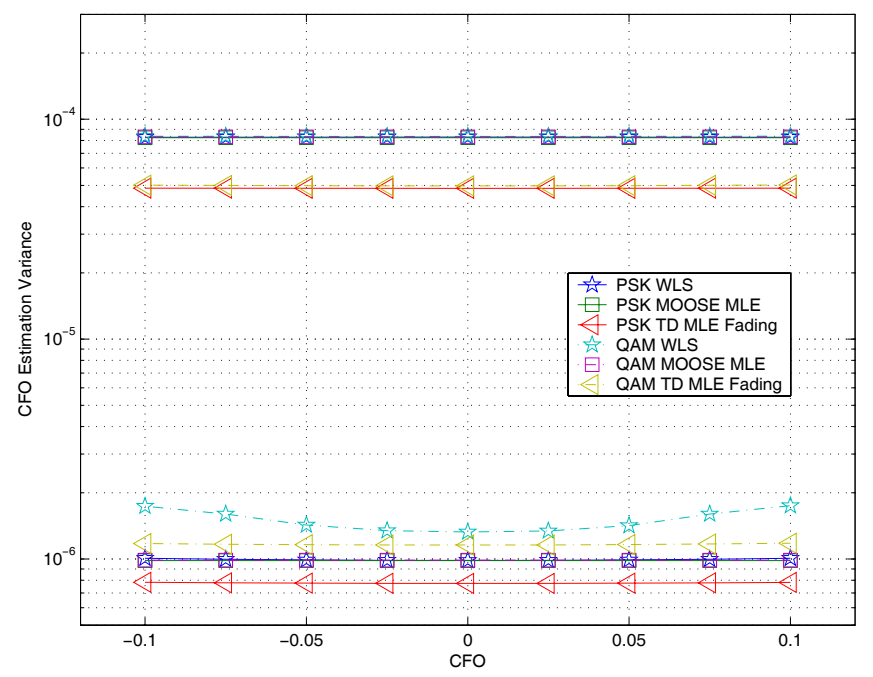

Fig. 9. Performance of various $\mathrm{CFO}$ estimation schemes under different CFO levels: time-varying fading channels and $\mathrm{SNR}=24 \mathrm{~dB}$.

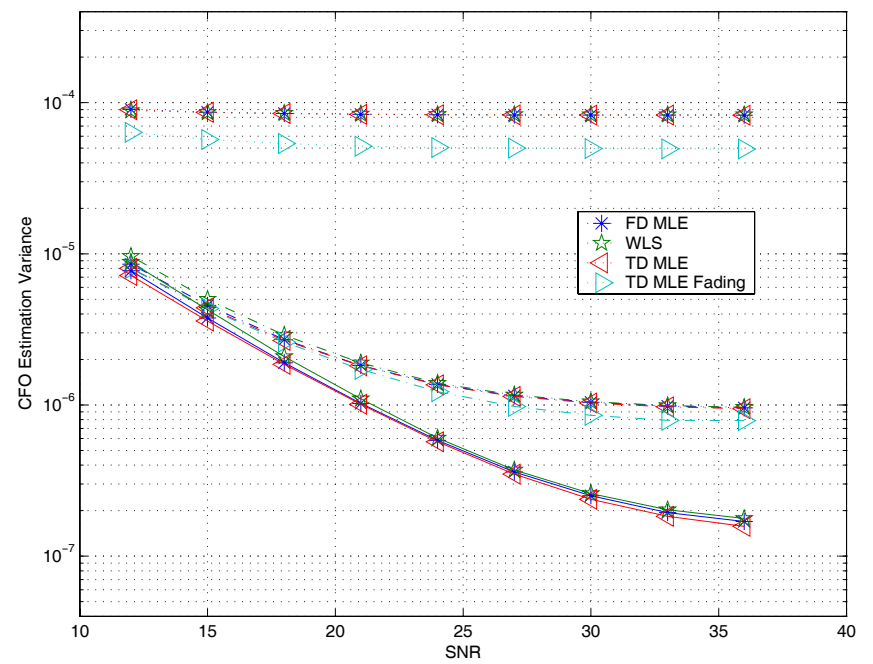

Fig. 10. Performance comparison of various CFO estimation schemes under static and slow/fast fading channel conditions: different QAM pilot symbols.

that, for the slow fading case, PSK WLS, PSK Moose MLE and QAM Moose MLE curves are overlapping. For the fast fading case, PSK TD MLE and QAM TD MLE have similar results and perform better than all other curves which are overlapping in the figure. From these results, we can see that the performance of the proposed scheme is not sensitive to the actual value of the CFO.

An important feature of the proposed time-domain scheme is that it can also work with different successive QAM pilot symbols, while the Moose MLE [8] can not. Simulations for different successive QAM pilot symbols are carried out under static, slow and fast fading channels, and the results are shown in Fig. 10 for comparison. The lowest set of curves marked by '-' has three curves (FD MLE, WLS and TD MLE). These curves are for static channels. The middle set marked by '.' has four curves as listed in the legend. These four curves are for slow fading channels and has a performance floor around $10^{-6}$ at high SNR. The top set marked by ':' also has four curves as listed in the legend. They are for the fast fading case. The FD MLE, WLS and TD MLE curves are overlapping in this case. The FD MLE curves plotted in these figures are for the estimator based on the frequency domain channel estimates, which takes the form,

$$
\hat{\epsilon}_{F D-M L E}=\frac{1}{\alpha}\left(\angle\left[\underline{\hat{H}}_{i}^{H} \underline{\hat{H}}_{i+1}\right]\right)
$$

The proposed MLE scheme yields best performance, because it takes fading effect into account.

When the time-domain channel-estimate based receiver structure (Fig. 1) is applied in OFDM systems, the proposed ML CFO estimate also has considerably low complexity compared to other frequency-domain CFO estimates. Note that, since the time-domain channel estimates already exist in these receivers (for the purpose of channel estimation), we can simply utilize that information without incuring additional computation going from the frequency domain to time domain. Looking at the full pilot symbol setting in the simulations, since the number of independent channel multipaths is $L=12$ and the number of subcarriers is $N=512$, the complexity of the proposed ML CFO is $O(L)=O(12)$, while the complexity of other frequency-domain CFO estimates is $O(N)=O(512)$, a significant complexity reduction. Furthermore, the proposed scheme can be implemented with partial pilot symbol settings that has less restriction on OFDM pilot symbol design. More specifically, for partial-pilot systems, the pilot pattern can be designed to facilitate effective channel estimates [14], [17], [23]. Once successive channel estimates are transformed into time domain, their phase rotation can be used to calculate the CFO.

In summary, the proposed CFO estimator exhibits superior performance with flexible pilot design requirement and reduced computational complexity. It is applicable to carrier frequency estimation and also suitable for continuous carrier frequency tracking in wireless OFDM systems.

\section{CONCLUSION}

This paper presented a CFO estimation scheme based on time-domain channel estimates. Exploring a receiver structure with embedded time-domain channel estimates, the proposed method is an approximate ML CFO estimate utilizing the CFO information which is exhibited through phase rotation of estimated channel multipaths. The proposed ML CFO estimate is further extended to the case of time-varying fading channels. Theoretical analysis and numerical simulations have been provided to show that the proposed scheme has excellent performance with considerably reduced complexity. Furthermore, since the scheme is based on time-domain channel estimates, it has less restriction on the pilot patterns. The idea of using the time-domain channel estimates can be further extended for the CFO estimation in MIMO-OFDM systems.

\section{APPENDIX A}

Derivation of $f\left(\underline{\hat{h}}_{p_{i+1}} ; \epsilon \mid \underline{\hat{h}}_{p_{i}}\right)$ UNDER TIME-VARYING FADING $\overline{\mathrm{CH}} i \mathrm{NNELS}$

We derive the joint pdf of $\underline{\hat{h}}_{p}=\left[\begin{array}{ll}\hat{h}_{p}^{T} & \hat{h}_{p}^{T} \\ \underline{h}_{i+1}\end{array}\right]^{T}$ using (18) as follows. 


$$
\begin{aligned}
& E\left[\underline{\hat{h}}_{i}{\underline{\hat{h}_{p}}}_{i}{ }_{i}\right]=E\left[\underline{\hat{h}}_{i+1}{\underline{\hat{h}_{p}}}_{i+1}^{H}\right] \\
& =E\left[\mathbf{U}_{p}^{H} \mathbf{X}^{-1} \mathbf{D}_{1}^{\prime} \mathbf{X} \underline{H}_{i} \underline{H}_{i}^{H} \mathbf{X}^{H} \mathbf{D}_{1}^{\prime H}\left(\mathbf{X}^{-1}\right)^{H} \mathbf{U}_{p}\right] \\
& +E\left[\mathbf{U}_{p}^{H} \mathbf{X}^{-1} \mathbf{D}_{1}^{\prime} \underline{Z}_{i} \underline{Z}_{i}^{H} \mathbf{D}_{1}^{\prime H}\left(\mathbf{X}^{-1}\right)^{H} \mathbf{U}_{p}\right] \\
& \approx \Lambda_{p}+\frac{\beta}{\gamma} \mathbf{I} \\
& E\left[\underline{\hat{h}}_{i} \underline{\hat{h}}_{i+1}^{H}\right]=E\left[\underline{\hat{h}}_{i+1} \underline{\hat{h}}_{i}^{H}{ }_{i}^{H}\right. \\
& =E\left[\mathbf{U}_{p}^{H} \mathbf{X}^{-1} \mathbf{D}_{1}^{\prime} \mathbf{X} \underline{H}_{i} \underline{H}_{i+1}^{H} \mathbf{X}^{H} e^{-j \alpha \epsilon} \mathbf{D}_{1}^{\prime H}\left(\mathbf{X}^{-1}\right)^{H} \mathbf{U}_{p}\right] \\
& \approx a e^{-j \alpha \epsilon} \Lambda_{p}
\end{aligned}
$$

using the approximations $E\left[\mathbf{X}^{-1} \underline{Z}_{i} \underline{Z}_{i}^{H}\left(\mathbf{X}^{-1}\right)^{H}\right] \approx \frac{\beta}{\gamma} \mathbf{I}$ and $\mathbf{D}_{1}^{\prime} \approx \mathbf{I}$. Therefore, we can derive the joint pdf of $\underline{\hat{h}_{p}} \sim$ $\mathcal{C N}(\underline{0}, \boldsymbol{\Sigma})$, where

$$
\boldsymbol{\Sigma}=\left[\begin{array}{l|l}
\boldsymbol{\Sigma}_{11} & \boldsymbol{\Sigma}_{12} \\
\hline \boldsymbol{\Sigma}_{21} & \boldsymbol{\Sigma}_{22}
\end{array}\right]
$$

with $\boldsymbol{\Sigma}_{11}=\boldsymbol{\Sigma}_{22} \approx \Lambda_{p}+\frac{\beta}{\gamma} \mathbf{I}$, and $\boldsymbol{\Sigma}_{12}=\boldsymbol{\Sigma}_{21}^{H} \approx$ $a e^{-j \alpha \epsilon} \Lambda_{p}$. Further derivation yields the conditional pdf of $f\left(\underline{\hat{h}}_{i+1} ; \epsilon \mid \underline{\hat{h}}_{i}\right)$ as

$$
\begin{array}{r}
f\left(\underline{\hat{h}}_{i+1} ; \epsilon \mid \underline{\hat{h}}_{i}\right)=c \cdot \exp \left\{-\frac{1}{2}\left(\underline{\hat{h}}_{\underline{p}}-\boldsymbol{\Sigma}_{21} \boldsymbol{\Sigma}_{11}^{-1} \underline{\hat{h}}_{\underline{p}}\right)^{H}\right. \\
\left.\times\left[\boldsymbol{\Sigma}_{22}-\boldsymbol{\Sigma}_{21} \boldsymbol{\Sigma}_{11}^{-1} \boldsymbol{\Sigma}_{12}\right]^{-1}\left(\underline{\hat{h}}_{i+1}-\boldsymbol{\Sigma}_{21} \boldsymbol{\Sigma}_{11}^{-1} \underline{\hat{h}}_{i}\right)\right\}
\end{array}
$$

This actually shows that $f\left(\hat{h}_{p_{i+1}} ; \epsilon \mid \underline{\hat{h}}_{i}\right)$ is Gaussian distributed with mean and covariance as

$$
\begin{aligned}
& E\left[\underline{\underline{h}}_{i+1} \mid \underline{\hat{h}_{p}}\right]=\boldsymbol{\Sigma}_{21} \boldsymbol{\Sigma}_{11}^{-1} \\
& \approx e^{j \alpha \epsilon} a \Lambda_{p}\left(\Lambda_{p}+\frac{\beta}{\gamma} \mathbf{I}\right)^{-1} \underline{\hat{h}}_{p_{i}} \approx e^{j \alpha \epsilon} \underline{h}_{p_{i}} \\
\mathbf{C}_{{\underline{h_{p}}}_{i+1} \mid \underline{h}_{p}} & =\boldsymbol{\Sigma}_{22}-\boldsymbol{\Sigma}_{21} \boldsymbol{\Sigma}_{11}^{-1} \boldsymbol{\Sigma}_{12} \\
& \approx\left(\Lambda_{p}+\frac{\beta}{\gamma} \mathbf{I}\right)-a^{2} \Lambda_{p}^{2}\left(\Lambda_{p}+\frac{\beta}{\gamma} \mathbf{I}\right)^{-1} \\
& =\left(\Lambda_{p}+\frac{\beta}{\gamma} \mathbf{I}\right)-a^{2} \Lambda_{p}\left[\mathbf{I}-\frac{\beta}{\gamma} \Lambda_{p}^{-1}+\left(\frac{\beta}{\gamma} \Lambda_{p}^{-1}\right)^{2} \cdots\right] \\
\{\gamma \gg 0\} & \approx\left(\Lambda_{p}+\frac{\beta}{\gamma} \mathbf{I}\right)-a^{2} \Lambda_{p}\left(\mathbf{I}-\frac{\beta}{\gamma} \Lambda_{p}^{-1}\right) \\
\{a \approx 1\} & \approx \frac{2 \beta}{\gamma}\left(\frac{\gamma(1-a)}{\beta} \Lambda_{p}+\mathbf{I}\right)
\end{aligned}
$$

\section{APPENDIX B}

\section{DERIVATION FOR THE CRLB UNDER TIME-VARYING}

\section{FADING CHANNELS}

When the CFO $\epsilon$ is small, by the assumption $\mathbf{D}_{1}^{\prime}=\mathbf{I}_{N \times N}$, $\mathbf{C}_{\underline{Y}}$ can be simplified as

$$
\mathbf{C}_{\underline{Y}}=\left[\begin{array}{c|c}
\mathbf{P}+N \sigma^{2} \mathbf{I} & a e^{-j \alpha \epsilon} \mathbf{P} \\
\hline a e^{j \alpha \epsilon} \mathbf{P} & \mathbf{P}+N \sigma^{2} \mathbf{I}
\end{array}\right]=\left[\begin{array}{c|c}
\mathbf{A} & \mathbf{B} \\
\hline \mathbf{C} & \mathbf{D}
\end{array}\right]
$$

Thus,

$$
\begin{aligned}
\frac{\partial \mathbf{C}_{\underline{Y}}}{\partial \epsilon} & =\left[\begin{array}{c|c}
\mathbf{0} & -j \alpha a e^{-j \alpha \epsilon} \mathbf{P} \\
\hline j \alpha a e^{j \alpha \epsilon} \mathbf{P} & \mathbf{0}
\end{array}\right] \\
& =\mathbf{Q C}_{\underline{Y}}-\mathbf{C}_{\underline{Y}} \mathbf{Q}, \quad \text { where } \mathbf{Q}=\left[\begin{array}{c|c}
\mathbf{0} & \mathbf{0} \\
\hline \mathbf{0} & j \alpha \mathbf{I}
\end{array}\right](80)
\end{aligned}
$$

The Fisher information matrix is then

$\mathbf{F I M}_{\epsilon}=\operatorname{tr}\left\{\mathbf{C}_{\underline{Y}}^{-1} \frac{\partial \mathbf{C}_{\underline{Y}}}{\partial \epsilon} \mathbf{C}_{\underline{Y}}^{-1} \frac{\partial \mathbf{C}_{\underline{Y}}}{\partial \epsilon}\right\}=2 \cdot \operatorname{tr}\left(\mathbf{Q Q}-\mathbf{Q C}_{\underline{Y}}^{-1} \mathbf{Q C}_{\underline{Y}}\right)$ where, we have used the property of the trace that $\operatorname{tr}(\mathbf{J K})=$ $\operatorname{tr}(\mathbf{K J})$. After some derivation, we can get

$$
\mathbf{F I M}_{\epsilon}=2 \alpha^{2} \cdot \operatorname{tr}\left\{\mathbf{D}^{-1} \mathbf{C S}_{\mathbf{c}}{ }^{-1} \mathbf{B}\right\}
$$

where, $\mathbf{S}_{\mathbf{c}}=\mathbf{A}-\mathbf{B D}^{-1} \mathbf{C}$ is the Schur's complement in the inversion of block matrix $\mathbf{C}_{\underline{Y}}$. The trace inside the Fisher information matrix is

$$
\begin{aligned}
& \operatorname{tr}\left\{\mathbf{D}^{-1} \mathbf{C S}_{\mathbf{c}}{ }^{-1} \mathbf{B}\right)=\operatorname{tr}\left\{\mathbf{B D} \mathbf{D}^{-1} \mathbf{C S}_{\mathbf{c}}{ }^{-1}\right\}=\operatorname{tr}\left\{\mathbf{A S}_{\mathbf{c}}{ }^{-1}-\mathbf{I}\right\} \\
& \text { where, } \mathbf{A}=\mathbf{P}+N \sigma^{2} \mathbf{I}=\mathbf{X} \mathbf{U} \Lambda \mathbf{U}^{H} \mathbf{X}^{H}+N \sigma^{2} \mathbf{I}
\end{aligned}
$$

We can further simplify the trace expression under the simplifying assumption that the signal constellation has a constant envelope like BPSK, 4-QAM, etc in which case, $\mathbf{X}^{-1}=\mathbf{X}^{H}$.

$$
\begin{aligned}
& \mathbf{A}=\mathbf{D}=\mathbf{X} \mathbf{U}\left(\Lambda+N \sigma^{2} \mathbf{I}\right) \mathbf{U}^{H} \mathbf{X}^{H}, \\
& \mathbf{A}^{-1}=\mathbf{D}^{-1}=\mathbf{X U}\left(\Lambda+N \sigma^{2} \mathbf{I}\right)^{-1} \mathbf{U}^{H} \mathbf{X}^{H},
\end{aligned}
$$

and $\quad \mathbf{S}_{\mathbf{c}}=\mathbf{X} \mathbf{U}\left[\Lambda+N \sigma^{2} \mathbf{I}-a^{2} \Lambda\left(\Lambda+N \sigma^{2} \mathbf{I}\right)^{-1} \Lambda\right] \mathbf{U}^{H} \mathbf{X}^{H}$

Hence,

$$
\mathbf{A S}_{\mathbf{c}}{ }^{-1}-\mathbf{I}=\mathbf{X} \mathbf{R}_{\underline{H H}}^{\prime} \mathbf{X}^{H}
$$

where, $\mathbf{R}_{\underline{H} H}^{\prime}=\mathbf{U}\left\{\left(\Lambda+N \sigma^{2} \mathbf{I}\right)\left[\Lambda+N \sigma^{2} \mathbf{I}-a^{2} \Lambda(\Lambda+\right.\right.$ $\left.\left.N \sigma^{2} \mathbf{I}\right)^{-1} \overline{\Lambda]^{-1}}-\mathbf{I}\right\} \mathbf{U}^{H}$. Thus, for constant envelope signals, the FIM can be expressed as

$$
\begin{aligned}
& \mathbf{F I M}_{\epsilon}=2 \alpha^{2} \operatorname{tr}\left\{\mathbf{A S}_{\mathbf{c}}{ }^{-1}-\mathbf{I}\right\}=2 \alpha^{2} \operatorname{tr}\left\{\mathbf{X R}_{\underline{H H}}^{\prime} \mathbf{X}^{H}\right\} \\
& =2 \alpha^{2} \sum_{i=0}^{N-1}\left|X_{i}\right|^{2} \mathbf{R}_{\underline{H H}}^{\prime}[i, i] \approx 2 \alpha^{2} E\left[\left|X_{i}\right|^{2}\right] \operatorname{tr}\left\{\underline{\mathbf{R}_{\underline{H}}^{\prime}}\right\}
\end{aligned}
$$

Even though the above derivation is based on constant envelope signals, fortunately, the exact (82) and the approximate (84) CRLBs almost exactly match, even for the non-constant envelope constellations. This is evident from Fig. 11 where the exact and approximate CRLBs for CFO estimation in fading channel with 64-QAM overlap on one another.

\section{APPENDIX C}

\section{Derivation of VARIANCE ANALysis For the Proposed} ML CFO ESTIMATORS

As discussed in Section, we consider a general channel model that accommodates both (19) and (20), which can be simply formulated as

$$
\underline{\hat{h}}_{i+1}=e^{j \alpha \epsilon} \underline{\hat{h}}_{i}+\underline{w}_{i+1}
$$

with $\underline{w}_{i+1} \sim \mathcal{C N}\left(\underline{0}, \mathbf{C}_{{\underline{\hat{h}_{p}}}_{\underline{p}_{1+1}}{\underline{\hat{h}_{p}}}_{i}}\right)$. Meanwhile, the derived CFO estimators have the general form as

$$
\hat{\epsilon}=\frac{1}{\alpha} \angle\left[\underline{\hat{h}}_{i}^{H} \mathbf{C}_{\underline{\hat{h}}_{p_{i+1}}}^{-1} \underline{\hat{h}}_{i} \underline{\hat{h}}_{i+1}\right]
$$

Considering the estimation error, it can be expressed as

$$
\begin{aligned}
& \hat{\epsilon}-\epsilon=\frac{1}{\alpha} \angle\left[\left(\underline{\hat{h}}_{p} e^{j \alpha \epsilon}\right)^{H} \mathbf{C}_{\hat{h}_{p_{i+1}}^{-1}}^{-1} \underline{\hat{h}}_{\underline{p}_{i}} \underline{\hat{h}}_{i+1}\right]
\end{aligned}
$$

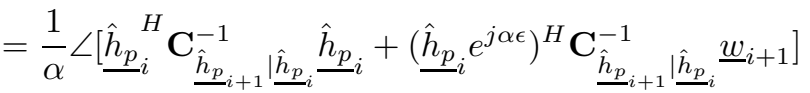




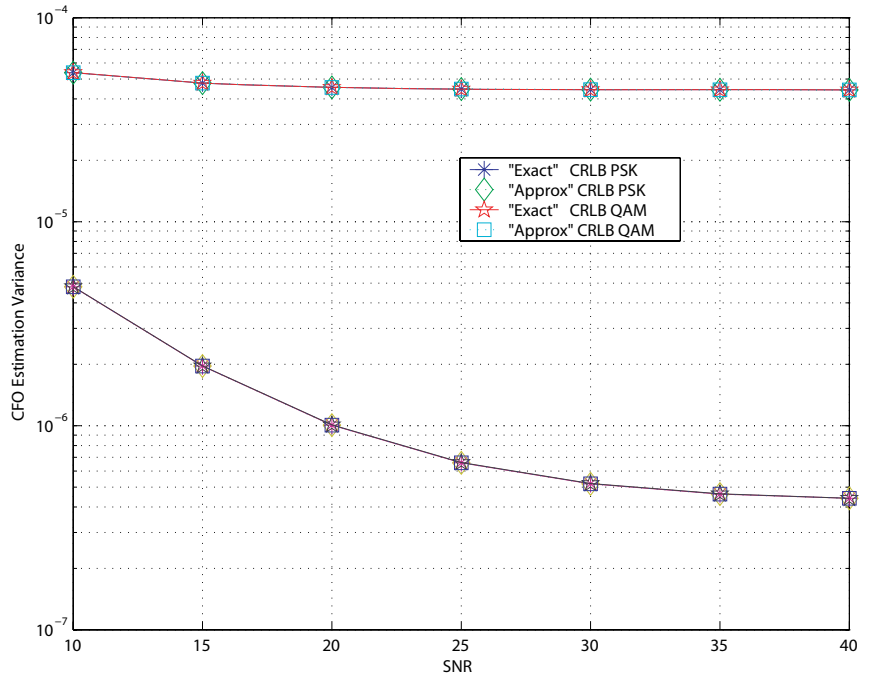

Fig. 11. Exact and approximate CRLBs for CFO estimation under fading channels (slow fading $F_{d}=0.005$ and fast fading $F_{d}=0.05$ ).

Note that, $\underline{\hat{h}}_{i}^{H} \mathbf{C}_{\underline{\hat{h}}_{p} i+1}^{-1} \underline{\underline{h}}_{\underline{i}} \underline{\hat{h}}_{p}$ is a real number. Under a high SNR and thus a small estimation error $\left(|\hat{\epsilon}-\epsilon| \ll \frac{1}{2 \pi}\right)$, the estimation error can be approximated by its tangent value as

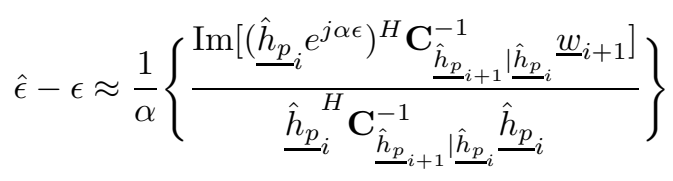

Case I. First look at the static channel, in which, ${\widehat{\mathbf{C}_{\hat{h}_{p}} \mid \hat{h}_{p}}}_{i} \approx \frac{2 \beta}{\gamma} \mathbf{I},(88)$ can be simply formulated at high SNRs as

$$
\begin{aligned}
\hat{\epsilon}-\epsilon & \approx \frac{1}{\alpha}\left\{\frac{\operatorname{Im}\left[\left(\underline{\hat{h}}_{i} e^{j \alpha \epsilon}\right)^{H} \underline{w}_{i+1}\right]}{\hat{h}_{p}^{H} \hat{h}_{p}}\right\} \\
& \approx \frac{1}{\alpha}\left\{\frac{\operatorname{Im}\left[\left(\underline{h}_{p} e^{j \alpha \epsilon}\right)^{H} \underline{w}_{i+1}\right]}{\underline{h}_{p}^{H}{ }^{H} \underline{h}_{p}}\right\}
\end{aligned}
$$

The variance of the estimation error is

$$
\begin{aligned}
\operatorname{var}(\hat{\epsilon}-\epsilon) & \approx \frac{1}{\alpha^{2}} \cdot \frac{\operatorname{var}\left(\operatorname{Im}\left[\left(\underline{h}_{p} e^{j \alpha \epsilon}\right)^{H} \underline{w}_{i+1}\right] \mid \underline{h}_{i}\right)}{\left|\underline{h}_{p}{ }_{i} \underline{h}_{p_{i}}\right|^{2}} \\
& =\frac{1}{2 \alpha^{2}} \cdot \frac{\frac{h_{p}{ }_{i}^{H}}{\mathbf{C}_{\hat{h}_{p}}} \underline{\underline{h}}_{i+1}{\underline{h_{p}}}_{i} \underline{h}_{i}}{\left|\underline{h}_{p}^{H} \underline{h}_{p}\right|^{2}} \\
& \approx \frac{1}{\alpha^{2}} \cdot \frac{\beta}{\gamma} \cdot \frac{1}{{\underline{h_{p}}}_{i}^{H} \underline{h}_{p}} \approx \frac{1}{\alpha^{2}} \cdot \frac{\beta}{N \gamma}
\end{aligned}
$$

where, we approximate the $\underline{h}_{p_{i}}{ }_{i}^{H} \underline{h}_{p}$ with its expectation, which is normalized to $N$ in this paper.

$\underline{\text { Case II }}$. As for the time-varying fading channel, $\mathbf{C}_{{\underline{\hat{h}_{p}}}_{i+1} \mid \underline{\hat{h}}_{\underline{i}}} \approx$ $\frac{2 \beta}{\gamma}\left(\frac{\gamma(1-a)}{\beta} \Lambda_{p}+\mathbf{I}\right)$, at high SNR, (88) can be simplified as

$$
\begin{aligned}
& \hat{\epsilon}-\epsilon \approx \frac{1}{\alpha}\left\{\frac{\operatorname{Im}\left[\left(\underline{h}_{p} e^{j \alpha \epsilon}\right)^{H} \mathbf{C}_{{\underline{h_{p}}}_{i+1}}^{-1}{\underline{h_{p}}}_{i} \underline{w}_{i+1}\right]}{\underline{h p}_{i}^{H} \mathbf{C}_{\hat{h}_{p}}^{-1} \underline{\underline{h}}_{i+1}{\underline{h_{p}}}_{i} \underline{h}_{p}}\right\} \\
& =\frac{1}{\alpha}\left\{\frac{\operatorname{Im}\left[\left(\underline{h}_{p_{i}} e^{j \alpha \epsilon}\right)^{H}\left(\frac{2 \beta}{\gamma}\left(\frac{\gamma(1-a)}{\beta} \Lambda_{p}+\mathbf{I}\right)\right)^{-1} \underline{w}_{i+1}\right]}{{\underline{h_{p}}}_{i}^{H}\left(\frac{2 \beta}{\gamma}\left(\frac{\gamma(1-a)}{\beta} \Lambda_{p}+\mathbf{I}\right)\right)^{-1} \underline{h}_{i}}\right\}
\end{aligned}
$$

The conditional variance of the estimation error can be expressed as

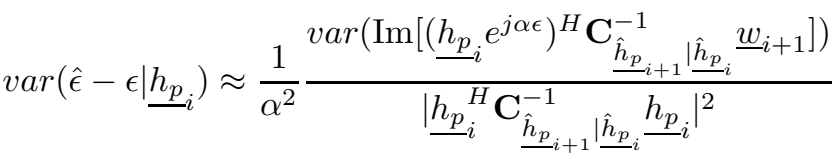

$$
\begin{aligned}
& =\frac{1}{2 \alpha^{2}} \frac{1}{{\underline{h_{p}}}_{i}^{H} \mathbf{C}_{{\underline{h_{p}}}_{i+1} \underline{h_{\underline{h}}}-1} \frac{h_{p}}{i}} \\
& \approx \frac{1}{\alpha^{2}} \cdot \frac{\beta}{\gamma} \cdot \frac{1}{\sum_{k=1}^{p} \frac{\left|h_{k, i}\right|^{2}}{\frac{\gamma}{\beta}(1-a) \lambda_{k}+1}}
\end{aligned}
$$

The estimator error variance is then approximated as

$$
\operatorname{var}(\hat{\epsilon}-\epsilon) \approx \frac{1}{\alpha^{2}} \cdot \frac{\beta}{\gamma \sum_{k=1}^{p} \frac{\lambda_{k}}{\frac{\gamma}{\beta}(1-a) \lambda_{k}+1}}
$$

Considering the equal-weight estimator under the timevarying fading channel, the estimation error would be

$$
\hat{\epsilon}-\epsilon \approx \frac{1}{\alpha}\left\{\frac{\operatorname{Im}\left[\left(\underline{h}_{p} e^{j \alpha \epsilon}\right)^{H} \underline{w}_{i+1}\right]}{{\underline{h_{p}}}_{i}^{H} \underline{h}_{i}}\right\}
$$

Its conditional variance can be expressed accordingly as

$$
\begin{array}{r}
\operatorname{var}\left(\hat{\epsilon}-\epsilon \mid \underline{h}_{i}\right) \approx \frac{1}{2 \alpha^{2}} \frac{{\underline{h_{p}}}_{i}^{H} E\left[\underline{w}_{i+1} \underline{w}_{i+1}^{H}\right] \underline{h_{p}}}{\left|\underline{h}_{p}^{H}{ }_{i} \underline{h p}_{i}\right|^{2}} \\
=\frac{1}{\alpha^{2}} \cdot \frac{\beta}{\gamma} \cdot \frac{\sum_{k=1}^{p}\left|h_{k, i}\right|^{2}\left(\frac{\gamma(1-a)}{\beta} \lambda_{k}+1\right)}{\left|\underline{h}_{i}{ }_{i}^{H} \underline{h}_{p}\right|^{2}}
\end{array}
$$

and its estimator error variance is approximated by

$$
\operatorname{var}(\hat{\epsilon}-\epsilon) \approx \frac{1}{\alpha^{2}} \cdot \frac{N \beta+\sum_{k=1}^{p} \lambda_{k}^{2} \gamma(1-a)}{\gamma N^{2}}
$$

\section{REFERENCES}

[1] H. Zhou, A. V. Malipatil and Y. F. Huang, "Maximum-Likelihood Carrier Frequency Offset Estimation for OFDM Systems in Fading Channels," IEEE WCNC, Apr 2006.

[2] J. A. C. Bingham, "Multicarrier modulation for data transmission: an idea whose time has come," in IEEE Trans. on Comm., vol. 28, no. 5, pp. 5-14, May 1990.

[3] L. Hanzo, M. Munster, B. J. Choi and T. Keller, OFDM and MC-CDMA for Broadband Multi-User Communications, WLANs and Broadcasting, IEEE Press, 2003.

[4] X. Ma, H. Kobayashi, S.C Schwartz, "Effect of frequency offset on BER of OFDM and single carrier systems," in IEEE International Conference on Communications, vol. 3, pp. 22-27, June 1996.

[5] C. R. N. Athaudage, "BER sensitivity of OFDM systems to time synchronization error," ICCS 2002., vol. 1, pp. 42-46, Nov. 2002.

[6] T. Pollet, M. V. Bladel, and M. Moeneclaey, "BER Sensitivity of OFDM Systems to Carrier Frequency Offset and Wiener Phase Noise," IEEE Trans. Comm., vol. 43, no. 2/3/4, pp. 191-193, Feb/Mar/Apr 1995.

[7] T. M. Schmidl and D. C. Cox, "Robust Frequency and Timing Synchronization for OFDM," IEEE Transactions on Communications, vol. 45, no. 12, pp. 1613 - 1621, Dec. 1997. 
[8] Paul H. Moose, “ A Technique for Orthogonal Frequency Division Multiplexing Frequency Offset Correction," IEEE Trans. Comm., vol. 42, no. 10, pp. 2908-2914, Oct. 1994.

[9] M. Speth, S. Fechtel, G. Fock, and H. Meyr, "Optimum Receiver Design for Wireless Broad-Band Systems Using OFDM - Part I," in IEEE Trans. on Comm., vol. 47, no. 11, pp. 1668 - 1677, Nov. 1999.

[10] A. J. Coulson, "Maximum likelihood synchronization for OFDM using a pilot symbol: algorithms," in IEEE Journal on Selected Areas in Communications., vol. 19, no. 12, pp. 2486 - 2494, Dec. 2001.

[11] Jungwon Lee, Hui-Ling Lou, D. Toumpakaris, J. M. Cioffi, "Effect of carrier frequency offset on OFDM systems for multipath fading channels," in Proc. IEEE GLOBECOMM'04, vol. 6, pp. 3721-3725, Nov 2004.

[12] L. Rugini, P. Banelli, "BER of OFDM Systems Impaired by Carrier Frequency Offset in Multipath Fading Channels," IEEE Trans. Wireless Comm., vol. 4, no. 5, pp. 2279-2288, Sept. 2005.

[13] H. Minn, V. K. Bhargava, and K. B. Letaief, "A Robust Timing and Frequency Synchronization for OFDM Systems," in IEEE Trans. on Wireless Comm., vol. 2, no. 4, pp. 822 - 839, July. 2003.

[14] S. Kapoor, D. J. Marchok and Y. F. Huang, " Pilot Assisted Synchronization for Wireless OFDM Systems over Fast Time Varying Fading Channels," IEEE VTC 98, 48th IEEE, vol. 3, pp. 2077 - 2080, May 1998.

[15] P. Y. Tsai, H. Y. Kang and T. D. Chiueh, "Joint Weighted LeastSquares Estimation of Carrier-Frequency Offset and Timing Offset for OFDM Systems Over Multipath Fading Channels," in IEEE Trans. on Veh. Technol., vol. 54, no. 1, pp. 211-223, Jan 2005.

[16] J.-J. van de Beek, O. Edfors, M. Sandell, S. K. Wilson and P. O. Borjesson, "On Channel Estimation in OFDM Systems," IEEE VTC 1995, July 1995, pp. 815-819.

[17] O. Edfors, M. Sandell, J.-J. van de Beek, S. K. Wilson and P. O. Borjesson, "OFDM Channel Estimation by Singular Value Decomposition," IEEE Trans. Comm.,, vol. 46, no. 7, pp. 931-939, July 1998.

[18] S. D. Silvey, Statistical Inference, London: Chapman and Hall, 1975

[19] Y. Li, L. J. Cimini, and N. R. Sollenberger, "Robust Channel Estimation for OFDM Systems with Rapid Dispersive Fading Channels," in IEEE Trans. on Comm., vol. 46, no. 7, pp. 902-915, July 1998.

[20] Y. R. Zheng and C. Xiao, "Simulation models with correct statistical properties for Rayleigh fading channels," in IEEE Trans. on Comm., vol. 51, no. 6, pp. 920 - 928, June. 2003.

[21] D. Schafhuber and G. Matz, "MMSE and Adaptive Prediction of TimeVarying Channels for OFDM Systems," IEEE Trans. on Wireless Comm., vol. 4, no. 2, pp. 593-602, 2005.

[22] Steven M. Kay, Fundamentals of statistical signal processing: estimation theory, Prentice-Hall, Inc., 1993.

[23] M. Speth, S. Fechtel, G. Fock and H. Meyr, "Optimum Receiver Design for OFDM-Based Broadband Transmission - Part II: A Case Study," in IEEE Trans. on Comm., vol. 49, no. 4, pp. 571 - 578, April. 2001.

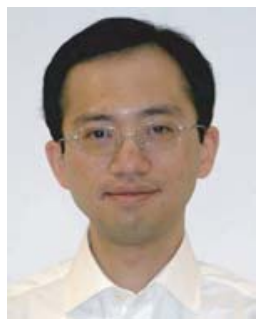

Hao Zhou was born in Zhejiang, China, in 1974. He received the B.S. and M.S. degrees in Electronic Engineering Department from SJTU, Shanghai, China, in 1997 and 2000. He studied at Univ. of Notre Dame, IN since 2001 and received the M.S. and $\mathrm{Ph} . \mathrm{D}$. degrees in the department of Electrical Engineering from Univ. of Notre Dame, in 2003 and 2007. From 2007, he is a senior signal processing engineer with Atheros Communications, Santa Clara, CA. His current research interests include mobile communications, satellite communications, modulation/demodulation, coding/decoding, and digital signal processing.

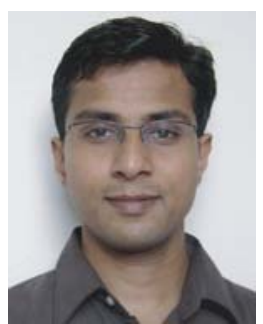

Amaresh V. Malipatil was born in Gulbarga, India. $\mathrm{He}$ received his Bachelor of Engineering degree in Electronics and Communications Engineering from National Institute of Technology Karnataka, India in 2001 and Master of Science in Electrical Engineering from the University of Notre Dame in 2005. He was a member of the Wireless LAN group at Hellosoft Inc. from 2001 to 2003, where he was involved in the development of $802.11 \mathrm{a} / \mathrm{b} / \mathrm{g}$ baseband modem solutions. Currently, he is a Serdes System Architect at LSI Corp. in Milpitas, CA, where he is working on design of high speed serial links. His research interests include linear and nonlinear signal processing with applications to communications, high speed serial interconnects, synchronization in OFDM systems and predistortion of high power amplifiers.

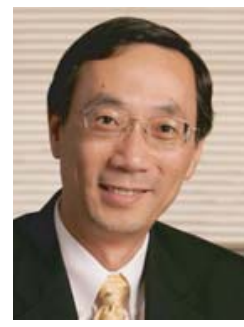

Yih-Fang Huang is Professor of the Department of Electrical Engineering at University of Notre Dame where he started as an assistant professor upon receiving his Ph.D in 1982, and he served as chair of the department from 1998 to 2006 In Spring 1993, Dr. Huang received the Toshiba Fellowship and was Toshiba Visiting Professor at Waseda University, Tokyo, Japan, in the Department of Electrical Engineering. From April to July 2007, he was a visiting professor at the Technical University of Munich. In Fall, 2007, Dr. Huang was awarded the Fulbright-Nokia scholarship for lectures/research at Helsinki University of Technology. Dr. Huangs research interests focus on statistical and adaptive signal processing. He has contributed to the field of Set-Membership Filtering (SMF), having developed a group of adaptive algorithms known as optimal bounding ellipsoids (OBE) algorithms. His recent interests are in applying statistical/adaptive signal processing to multiple-access wireless communication systems. Dr. Huang received the Golden Jubilee Medal of the IEEE Circuits and Systems Society in 1999, served as Vice President in 1997-98 and was a Distinguished Lecturer for the same society in 2000-2001. He received the University of Notre Dame's Presidential Award in 2003. Dr. Huang is a Fellow of the IEEE. 